\title{
M-theoretic Lichnerowicz formula and supersymmetry
}

\author{
André Coimbra $^{a}$ and Ruben Minasian ${ }^{b}$ \\ ${ }^{a}$ Institut des Hautes Etudes Scientifiques, Université Paris Saclay, \\ Le Bois-Marie 35 rte de Chartres, F-91440 Bures-sur-Yvette, France \\ ${ }^{b}$ Institut de Physique Théorique, Université Paris Saclay, CNRS, CEA, \\ Orme des Merisiers, F-91191 Gif-sur-Yvette, France \\ E-mail: coimbra@ihes.fr, ruben.minasian@ipht.fr
}

ABSTRACT: A suitable generalisation of the Lichnerowicz formula can relate the squares of supersymmetric operators to the effective action, the Bianchi identities for fluxes, and some equations of motion. Recently, such formulae have also been shown to underlie the (generalised) geometry of supersymmetric theories. In this paper, we derive an M-theoretic Lichnerowicz formula that describes eleven-dimensional supergravity together with its higherderivative couplings. The first corrections to the action appear at eight-derivative level, and the construction yields two different supersymmetric invariants, each with a free coefficient. We discuss the restriction of our construction to seven-dimensional internal spaces, and implications for compactifications on manifolds of $G_{2}$ holonomy. Inclusion of fluxes and computation of contributions with higher than eight derivatives are also discussed.

KeYwords: Flux compactifications, M-Theory, Supergravity Models

ARXIV EPRINT: 1705.04330 


\section{Contents}

1 Introduction 1

2 Eleven-dimensional supergravity 4

2.1 Review of higher-derivative terms 5

3 Lichnerowicz formula for eleven-dimensional supergravity $\quad 7$

$\begin{array}{lll}3.1 & \text { Lichnerowicz method } & 8\end{array}$

4 Higher-derivative terms $\quad 9$

$\begin{array}{lll}4.1 & R^{2} \text { and } R^{3} \text { couplings in the effective action } & 10\end{array}$

$\begin{array}{lll}4.2 & R^{4} \text { couplings } & 12\end{array}$

$\begin{array}{lll}\text { 4.2.1 Minimal solution } & 14\end{array}$

5 Application: seven-dimensional internal spaces $\quad 16$

$\begin{array}{lll}5.1 & \text { M-theory Lichnerowicz for } \mathrm{SO}(7) & 17\end{array}$

$\begin{array}{lll}\text { 5.1.1 Three-derivative terms in the operators } & 18\end{array}$

$\begin{array}{lll}5.1 .2 & \text { Five-derivative terms } & 18\end{array}$

$\begin{array}{lll}5.1 .3 & \text { Seven-derivative terms } & 19\end{array}$

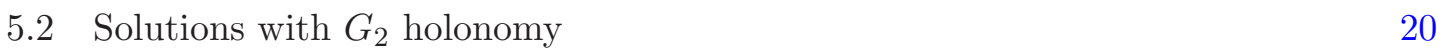

5.3 Comments on compactifications 22

6 Future directions $\quad 23$

6.1 Adding fluxes 24

6.2 Towards $R^{7} \quad 24$

A Projectors for $R^{3} \quad \mathbf{2 5}$

A.1 In eleven dimensions 25

$\begin{array}{ll}\text { A.2 In seven dimensions } & 29\end{array}$

$\begin{array}{ll}\text { B More general solution } & 31\end{array}$

\section{Introduction}

Recent development of generalised complex geometry [1,2] has been intimately related with our understanding of supersymmetric theories and has provided a natural language for classifying supersymmetric vacua and revealing the structure of off-shell supersymmetry in effective theories [3-8]. It is still, however, an open question whether generalised complex geometry can capture the structure of stringy perturbative corrections to the effective 
theories. If so one may hope that the geometric insight might one day translate into computational tools.

In its most basic formulation, GCG unites the geometric data with the antisymmetric two form field and puts diffeomorphisms and gerbe gauge transformations on equal footing [1]. This unified formalism allows a transfer of many results of Riemannian geometry to the generalised tangent bundle [2]. Notably, a generalised vanishing torsion condition on a generalised metric-connection yields a notion of a generalised Ricci tensor which fully captures the physics of the ten-dimensional NS sector - the action and the equations of motion [9]. Interestingly, and crucially for this work, the part covering only the metric and two-form field can alternatively be derived by using a version of the Lichnerowicz formula with closed three-form torsion $H$, due to Bismut. The standard Lichnerowicz formula [10] for a Levi-Civita connection states that the difference of the squares of the covariant derivative and the Dirac operator acts tensorially on a spinor and can serve as a definition of a scalar curvature $\mathcal{R}$ :

$$
\left.((\not))^{2}-\nabla^{a} \nabla_{a}\right) \varepsilon=-\frac{1}{4} \mathcal{R} \varepsilon .
$$

Here and throughout the paper $\nabla$ denotes the covariant derivative with respect to the torsion-free Levi-Civita connection. The Bismut version [11] finds a pair of operators with torsion such that the difference of their squares is again tensorial

$$
\left(\not^{H}\right)^{2} \varepsilon-\left(\tilde{\nabla}^{H}\right)^{a} \tilde{\nabla}_{a}^{H} \varepsilon=-\frac{1}{4} \mathcal{R} \varepsilon+\frac{1}{48} H^{2} \varepsilon,
$$

where

$$
\begin{aligned}
\tilde{\nabla}_{a}^{H} \varepsilon & =\nabla \epsilon+\frac{1}{8} H_{a b c} \gamma^{b c} \varepsilon, \\
\not^{H} \varepsilon & =\not \nabla \varepsilon+\frac{1}{24} H_{a b c} \gamma^{a b c} \varepsilon .
\end{aligned}
$$

Note that the Dirac operator is no longer the trace of the covariant derivative with torsionful connection! Also, strictly speaking, the tensor has two parts now - a scalar component given by a sum of the scalar curvature and $H^{2}$ and a four-form given by the (vanishing) exterior derivative of $H$.

Interestingly enough it is the inclusion of the dilaton that requires a truly generalised treatment. The key here is having a different action of the same operator on a spinor and a vector-spinor. The above-mentioned pair of operators is then seen as a component of the same "generalised Levi-Civita connection" $D$. This pair also appears in the respective supersymmetry variations of gravitino and dilatino

$$
\begin{aligned}
\delta \psi_{a} & =D_{a} \varepsilon=\nabla_{a} \epsilon+\frac{1}{8} H_{a \bar{b} \bar{c}} \gamma^{\bar{b} \bar{c}} \varepsilon \\
\delta \rho & =\gamma^{\bar{a}} D_{\bar{a}} \varepsilon=\gamma^{\bar{a}} \nabla_{\bar{a}} \varepsilon+\frac{1}{24} H_{\bar{a} \bar{b} \bar{c}} \gamma^{\bar{a} \bar{b} \bar{c}} \varepsilon-\gamma^{\bar{a}}\left(\partial_{\bar{a}} \phi\right) \varepsilon
\end{aligned}
$$

and the Lichnerowicz formula yields:

$$
\left((\not D)^{2}-D^{a} D_{a}\right) \varepsilon=-\frac{1}{4} S \varepsilon-\gamma^{a b c d} I_{a b c d} \varepsilon
$$


where on the right-hand side we find the bosonic NS action $S$, and the Bianchi identity $I_{a b c d}=\nabla_{[a} H_{b c d]}$.

The passage to non-closed torsion $H$ requires an extension of the generalised tangent bundle and the inclusion of gauge fields, eventually leading to a generalised complex description of heterotic strings. Once one properly extends the notion of covariant derivative to also cover gaugino variations, the Lichnerowicz theorem yields the ten-dimensional $N=1$ supergravity coupled to supersymmetric YM, and can further accommodate the heterotic Bianchi identity and the higher derivative terms in the effective action that serve its supersymmetry completion [12]. Interestingly, a single four-derivative correction to the Bianchi identity requires an infinity of terms in the supersymmetry variations and hence in the effective action $[13,14]$. Using the simple principle of building quadratic combinations of first order operators appearing in the supersymmetry variations that act only tensorially, allows one to compute the $\mathcal{O}\left(\alpha^{\prime 4}\right)[15]$.

It is also possible to fully geometrise both the NS and RR sector, or alternatively describe M-theory, of the internal $d$-dimensional space of a generic supersymmetric compactification in the language of exceptional generalised geometry, where the generalised tangent bundle corresponds to a representation of an exceptional $E_{d(d)}$ group [16, 17]. The bosonic action can again be written in the form of a Lichnerowicz equation for generalised Levi-Civita connections $[18,19]$. Schematically

$$
D \otimes_{S} D \otimes_{S} \varepsilon-D \otimes_{S} D \otimes_{J} \varepsilon=-\frac{1}{4} S_{\mathrm{B}} \varepsilon
$$

where $\otimes_{S}, \otimes_{J}$ denote projections to the generalised spinor bundles into which, respectively, the spinor and the gravitino embed, and now $S_{\mathrm{B}}$ is the full bosonic action, with RR fields, of the dimensionally restricted theory.

At this point one would like to dream that the suitable versions of the Lichnerowicz formula underlie every supersymmetric theory. Eleven-dimensional supergravity, even at a classical level, would appear to be the best candidate for undermining such a belief. Indeed the theory does not have a dilatino, and hence there is no natural Dirac operator other than the trace of the gravitino variation. Moreover, any Lichnerowicz type formula like (1.5) built out of operators linear in fields would yield an action that is at most quadratic. From other side in spite of its simplicity the eleven-dimensional supergravity action contains a cubic Chern-Simons interaction.

Yet, as we shall see, there is an appropriate generalisation. Indeed, using the LeviCivita connection and the four-form flux $G$ one may find an operator $\nabla_{a}^{G}$ such that it yields a tensorial polyform $\rho$ (there are no derivatives acting on $\varepsilon$ on the right-hand side) when squared:

$$
\gamma^{a b} \nabla_{a}^{G} \nabla_{b}^{G} \varepsilon=-\frac{1}{4} \rho \cdot \varepsilon
$$

Moreover requiring that $\rho$ has only an 11 -form (or scalar) and an 8 -form component ${ }^{1}$ uniquely fixes $\nabla_{a}^{G}$ to be the operator that appears in the gravitino variation $\delta \psi_{a}=\nabla_{a}^{G} \varepsilon$.

\footnotetext{
${ }^{1}$ We are dropping here the five-form which corresponds to the Bianchi identity $\nabla_{a} G_{b c d e} \gamma^{a b c d e}$.
} 
Furthermore, we may observe that up to integration by parts the Mukai pairing

$$
\mathcal{L}_{B}=\langle 1+C, \rho\rangle=\left.\rho\right|_{11}-\left.C \wedge \rho\right|_{8},
$$

gives the Lagrangian for eleven-dimensional supergravity.

Note that we have used very little here. As far as the generalised geometry constructions go, we seem to just pass to what locally looks like $T M \oplus \Lambda^{2} T^{*} M$, hence avoiding the complications of exceptional (and possibly infinite-dimensional) geometries, dual gravitons, need to stick to linearised equations and so on. Some or all of these issues arising in the full geometrisation of M-theory and U-duality groups will eventually have to be faced, and contrasted to the simplicity of (1.8). Instead of doing all this, we shall try to build on this construction, and modify $\nabla_{a}^{G}$ by higher-derivative contributions and verify if the method of requiring bilinears with tensorial action can reproduce the quantum higher-derivative corrections to M-theory.

The formula (1.7), where now the corrected operator $\nabla_{a}^{G}$ will a priori be all order in derivatives, is what we shall call M-theoretic Lichnerowicz formula. Actually we shall see that the first modifications from the one-derivative supergravity operator will start at seven derivatives, and the "minimal" version of the corrected operator can contain only $6 l+1$ derivatives (for integer $l$ ). When such operator is plugged into (1.8) it will clearly produce a series of terms in $\mathcal{L}_{B}$ with $2 \times(3 l+1)$ derivatives. $^{2}$ The use of the computer algebra system Cadabra $[20,21]$ was crucial to verify these computations.

The structure of the paper is as follows. In section 2, we review the eleven-dimensional supergravity and the first $\sim R^{4}$ quantum corrections. The M-theoretic Lichnerowicz formula is set up in section 3. Section 4 extends the construction to higher derivative terms, verifying that the first corrections appear at eight-derivative level, and constructing explicitly two different supersymmetric invariants (each coming with a free coefficient). We specialise on the case of seven-dimensional internal spaces in section 5. Possible extensions of our construction are discussed in section 6 .

\section{Eleven-dimensional supergravity}

Let us start by reviewing classical eleven-dimensional supergravity [22], to leading order in the fermions, following the conventions of $[18,19,23]$. The bosonic fields are the elevendimensional Lorentzian metric $g_{a b}$ and a three-form Abelian gauge potential $C_{a b c}$ and there is a fermionic gravitino field $\psi_{a}$.

Writing $\mathcal{R}$ for the Ricci scalar for the Levi-Civita connection $\nabla$ and $G=\mathrm{d} C$ for the four-form field-strength, the bosonic action is given by

$$
S_{\mathrm{B}}=\frac{1}{2 \kappa^{2}} \int\left(\mathcal{R} * 1-\frac{1}{2} G \wedge * G-\frac{1}{6} C \wedge G \wedge G\right),
$$

and taking $\gamma^{a}$ to be the $\operatorname{Cliff}(10,1 ; \mathbb{R})$ gamma matrices, the fermionic action is

$$
S_{\mathrm{F}}=\frac{1}{\kappa^{2}} \int \sqrt{-g}\left(\bar{\psi}_{a} \gamma^{a b c} \nabla_{b} \psi_{c}+\frac{1}{96} G_{a_{1} \ldots a_{4}} \bar{\psi}_{b}\left(\gamma^{a_{1} \ldots a_{4} b c}+12 \gamma^{a_{1} a_{2}} g^{a_{3} b} g^{a_{4} c}\right) \psi_{c}\right) .
$$

\footnotetext{
${ }^{2}$ Note that the opposite is not true. An action with $2 \times(3 l+1)$ derivatives does not necessarily restrict $\nabla_{a}^{G}$ to have only $(6 l+1)$-derivative terms.
} 
This leads to the equations of motion for the metric and gauge field

$$
\begin{aligned}
\mathcal{R}_{a b}-\frac{1}{12}\left(G_{a c_{1} c_{2} c_{3}} G_{b}{ }^{c_{1} c_{2} c_{3}}-\frac{1}{12} g_{a b} G^{2}\right) & =0, \\
\mathrm{~d} * G+\frac{1}{2} G \wedge G & =0,
\end{aligned}
$$

where $\mathcal{R}_{a b}$ is the Ricci tensor, and the gravitino equation of motion is

$$
\gamma^{a b c} \nabla_{b} \psi_{c}+\frac{1}{96}\left(G_{c_{1} \ldots c_{4}} \gamma^{a b c_{1} \ldots c_{4}}+12 G_{c_{1} c_{2}}^{a b} \gamma^{c_{1} c_{2}}\right) \psi_{b}=0
$$

This action is invariant under supersymmetry, with the transformation of the bosons given by

$$
\begin{aligned}
\delta g_{a b} & =2 \bar{\varepsilon} \gamma_{(a} \psi_{b)}, \\
\delta C_{a b c} & =-3 \bar{\varepsilon} \gamma_{[a b} \psi_{c]},
\end{aligned}
$$

while the supersymmetry variation of the gravitino is

$$
\delta \psi_{a}=\nabla_{a} \varepsilon+\frac{1}{288}\left(\gamma_{a}^{b_{1} \ldots b_{4}}-8 \delta_{a}^{b_{1}} \gamma^{b_{2} b_{3} b_{4}}\right) G_{b_{1} \ldots b_{4}} \varepsilon
$$

where $\varepsilon$ is the supersymmetry parameter.

\subsection{Review of higher-derivative terms}

In presence of M5 branes, this classical action is not consistent without the inclusion of certain higher-derivative terms. Indeed, since a single M5 supports a chiral six-dimensional $(2,0)$ tensor multiplet on the worldvolume, it is anomalous. The anomaly is a descendant of a particular eight-derivative eight-form polynomial. To cancel this anomaly via the inflow mechanism, one needs bulk couplings which in absence of M5 sources are invariant under diffeomorphisms and $C$-field gauge transformations, but become anomalous in the presence of the branes. Moreover this anomaly should restrict to the six-dimensional worldvolume.

The anomaly cancelling bulk term is of course only a part of the story, as it is hardly supersymmetric on its own. From one side it should receive contributions from $G$-flux. From other, it requires completion and other (non-anomalous) couplings with the same number of derivatives. Furthermore, since M-theory is supposed to be the strong coupling limit of type IIA strings, the eleven-dimensional couplings should also be seen in the decompactification limit of the string theory.

The eight-derivative couplings in string theory have a long history and (without fluxes) have been computed using string perturbation theory. In IIA there are only tree-level and one loop terms, while IIB also has D-instanton contributions. The tree level corrections are the same in both theories and take the form $[24,25]$

$$
e^{-1} \mathcal{L} \sim e^{-2 \phi}\left(t_{8} t_{8} R^{4}-\frac{1}{4} E_{8}\right)
$$

and the CP-even sector of the one-loop is given by [26]

$$
e^{-1} \mathcal{L}_{\mathrm{CP} \text {-even }} \sim\left(t_{8} t_{8} R^{4} \pm \frac{1}{4} E_{8}\right),
$$


where the top (bottom) sign is for the IIA (IIB) theory. The $E_{8}$ term can be written using two totally antisymmetric $\epsilon$-tensors. ${ }^{3}$ In addition, the IIA theory has a CP-odd one-loop term $\mathcal{L}_{\text {CP-odd }} \sim B_{2} \wedge\left[\operatorname{tr} R^{4}-\frac{1}{4}\left(\operatorname{tr} R^{2}\right)^{2}\right][28,29]$. Its lift to eleven dimensions

$$
\mathcal{L}_{11 \mathrm{~d}} \sim C_{3} \wedge\left[\operatorname{tr} R^{4}-\frac{1}{4}\left(\operatorname{tr} R^{2}\right)^{2}\right]
$$

cancels the M5 anomalies via inflow. The one-loop CP-even terms (2.8) also lift, while the tree-level contribution (2.7) is suppressed [30]. ${ }^{4}$

Our expectation is that the bosonic action of supersymmetric M-theory (just like any other supersymmetric theory) should be formulated as a kind of Lichnerowicz theorem. As a first step, we shall verify that this is the case for eleven-dimensional supergravity and recast the action as a Mukai transformed square of a first-order operator. Unsurprisingly, the operator in question turns out to be the covariant derivative with $G$-flux terms included that appears in the gravitino supersymmetry variation (2.6).

The modifications of this operator with higher-derivative terms included should in turn yield the supersymmetry variation for the M-theory action with higher-derivative terms included. Such supersymmetry modifications have been discussed e.g. in [31-33]; seven-derivative corrections were computed explicitly in [34,35] by reading them off from the effective action. Unfortunately, these are written in a different basis from ours, and due to the complicated nature of the terms the direct comparison of two results in not straightforward. For special cases of seven and eight-dimensions (for manifolds of $G_{2}$ and Spin(7) holonomy), simplified transformations have been suggested in [36].

The potential usefulness of the explicit form of the transformations in a convenient basis for e.g. compactifications makes our computation worthwhile. Our main motivation has been to test the Lichnerowicz method, and the hypothesis that it should underlie any supersymmetric theory. We also hope that this will provide a better systematics for still open questions such as inclusion of fluxes (which is very hard even in string theory, due to the need to perform higher-point calculations), or higher derivative terms (which in string theory, for the terms that appear in eleven dimensions, would also correspond to going to higher string loops). Finally, establishing an M-theoretic Lichnerowicz formula, analogous to the string theoretic cousins that have a generalised geometric origin, will hopefully lead to new insights about the geometry of M-theory.

${ }^{3}$ We follow the $R^{4}$ conventions of [27] and define

$$
t_{8} t_{8} R^{4}=t_{a_{1} \cdots a_{8}} t_{b_{1} \cdots b_{8}} R_{b_{1} b_{2}}^{a_{1} a_{2}} R_{b_{3} b_{4}}^{a_{3} a_{4}} R_{b_{5} b_{6}}^{a_{5} a_{6}} R_{b_{7} b_{8}}^{a_{7} a_{8}}
$$

and

$$
E_{8}=8 ! \times \delta_{a_{1} \cdots a_{8}}^{b_{1} \cdots b_{8}} R_{b_{1} b_{2}}^{a_{1} a_{2}} R_{b_{3} b_{4}}^{a_{3} a_{4}} R_{b_{5} b_{6}}^{a_{5} a_{6}} R^{a_{7} a_{8}}{ }_{b_{7} b_{8}}
$$

Note that for any antisymmetric matrix $M, t_{8} M^{4}=24\left(\operatorname{tr} M^{4}-\frac{1}{4}\left(\operatorname{tr} M^{2}\right)^{2}\right)$, and there is a useful relation between two quantities given by $\frac{1}{4} E_{8}=t_{8} t_{8} R^{4}+192 \operatorname{tr} R_{a b} R_{c d} \operatorname{tr} R_{a c} R_{b d}-768 \operatorname{tr} R^{a b c d} R_{a}{ }^{e}{ }_{c}^{f} R_{e}{ }^{g}{ }_{b}{ }^{h} R_{f g d h}+$ Ricci terms.

${ }^{4}$ The string $l$-loop terms surviving in the eleven-dimensional limit are $\sim R^{3 l+1}$. Note that due to the relation to anomalies, the one loop term is not renormalised, and the terms with a number of derivative higher than eight should not contain any top-form couplings of the type (2.9). 


\section{Lichnerowicz formula for eleven-dimensional supergravity}

Let us write the operator that appears in the gravitino variation as

$$
\delta \psi_{a}=\nabla_{a} \varepsilon+\frac{1}{288}\left(\gamma_{a}^{b_{1} \ldots b_{4}}-8 \delta_{b}^{b_{1}} \gamma^{b_{2} b_{3} b_{4}}\right) G_{b_{1} \ldots b_{4}} \varepsilon=\nabla_{a}^{G} \varepsilon
$$

It has long been known (see for example [23]) that there is an "integrability" condition satisfied by this connection

$$
\gamma^{a}\left[\nabla_{a}^{G}, \nabla_{b}^{G}\right] \varepsilon \propto(\text { all bosonic eoms })_{b} \cdot \varepsilon
$$

We can view this as being a consequence of supersymmetry. If we also denote the M-theory Rarita-Schwinger operator in the gravitino equation of motion,

$$
\gamma^{a b c} \nabla_{b} \psi_{c}+\frac{1}{96}\left(G_{c_{1} \ldots c_{4}} \gamma^{a b c_{1} \ldots c_{4}}+12 G_{c_{1} c_{2}}^{a b} \gamma^{c_{1} c_{2}}\right) \psi_{b}=L^{a b} \psi_{b}
$$

we observe that it such that $L^{a b} \psi_{b}=\gamma^{a b c} \nabla_{b}^{G} \psi_{c}$, and so by applying a supersymmetry transformation

$(\text { bosonic eoms })^{a} \cdot \varepsilon \propto \delta_{\varepsilon}(\text { fermionic eoms })^{a}=\delta_{\varepsilon}\left(L^{a b} \psi_{b}\right)=\delta_{\varepsilon}\left(\gamma^{a b c} \nabla_{b}^{G} \psi_{c}\right)=\gamma^{a b c} \nabla_{b}^{G} \nabla_{c}^{G} \varepsilon$

which implies (3.2), as was remarked, for instance, in [37]. It was also noted in [37] that if the equations of motion are solved then $L \circ \nabla^{G}=0$. Therefore, when on-shell we have an exact sequence

$$
S \stackrel{\nabla^{G}}{\longrightarrow} T^{*} \otimes S \stackrel{L}{\rightarrow} T^{*} \otimes S \stackrel{\left(\nabla^{G}\right)^{\dagger}}{\longrightarrow} S .
$$

Here we write $S$ and $T^{*}$ for the spinor and cotangent bundles respectively and have used $L=L^{\dagger} \Rightarrow\left(\nabla^{G}\right)^{\dagger} \circ L=0$. As we just saw, the condition $L \circ \nabla^{G}=0$ results from supersymmetry, while $L=L^{\dagger}$ can be derived from requiring the reality of $\int \bar{\psi}_{a} L^{a b} \psi_{b}$.

What appears to be less know is that if we write $\left(\tilde{\nabla}^{G}\right)^{c}=\frac{1}{9} \gamma_{a} L^{a c}=\gamma^{b c} \nabla_{b}^{G}$, i.e. the (left) gamma trace of the M-theory Rarita-Schwinger operator, then it is possible to recover the bosonic action from a Lichnerowicz-type relation $\left(\tilde{\nabla}^{G}\right)^{a} \nabla_{a}^{G} \varepsilon \sim \mathcal{L}_{B} \varepsilon$. More exactly, from (3.4) we see that $\tilde{\nabla}^{G} \nabla^{G}$ will be proportional to part of the bosonic equations of motion $\gamma^{a b} \nabla_{a}^{G} \nabla_{b}^{G} \varepsilon \propto$ (trace of Einstein equation +8 -form gauge equation) which can be used to reconstruct the bosonic action since $\mathcal{L}_{B} \sim\left(\right.$ trace of Einstein) $\operatorname{vol}_{g}+C \wedge(8$-form), up to integration by parts.

Explicitly we have

$$
\begin{aligned}
\nabla^{G}: S & \rightarrow T^{*} \otimes S, \\
\nabla_{a}^{G} \varepsilon & =\nabla_{a} \varepsilon+\frac{1}{288}\left(\gamma_{a}^{b_{1} \ldots b_{4}}-8 \delta_{a}^{b_{1}} \gamma^{b_{2} b_{3} b_{4}}\right) G_{b_{1} \ldots b_{4}} \varepsilon, \\
\tilde{\nabla}^{G}: T^{*} \otimes S & \rightarrow S \\
\tilde{\nabla}_{a}^{G} \psi^{a} & =\gamma^{a b} \nabla_{a} \psi_{b}+\frac{1}{144}\left(\gamma^{a b_{1} \ldots b_{4}}-2 \delta_{a}{ }^{b_{1}} \gamma^{b_{2} b_{3} b_{4}}\right) G_{b_{1} \ldots b_{4}} \psi_{a},
\end{aligned}
$$


and so $\tilde{\nabla}^{G}$ is the gamma-trace of gravitino equation of motion, i.e. it appears in the action in the term $\int \bar{\psi}\left(\tilde{\nabla}^{G}\right)^{a} \psi_{a}$. Note also that $L=L^{\dagger} \Rightarrow\left(\tilde{\nabla}^{G}\right)^{a} \gamma_{a}=\gamma_{a}\left(\left(\tilde{\nabla}^{G}\right)^{\dagger}\right)^{a}$. Then the Lichnerowicz relation is

$$
\begin{aligned}
\left(\tilde{\nabla}^{G}\right)^{a} \nabla_{a}^{G} \varepsilon= & -\frac{1}{4} \mathcal{R} \varepsilon+\frac{1}{24} \frac{1}{4 !} G_{b_{1} \ldots b_{4}} G^{b_{1} \ldots b_{4}} \varepsilon-\frac{1}{144}\left(\nabla_{b_{1}} G_{b_{2} \ldots b_{5}}\right) \gamma^{b_{1} \ldots b_{5}} \varepsilon \\
& +\frac{1}{4} \frac{1}{3} \frac{1}{3 !}\left(\nabla^{b_{1}} G_{b_{1} \ldots b_{4}}\right) \gamma^{b_{2} b_{3} b_{4}} \varepsilon-\frac{1}{4} \frac{1}{6} \frac{1}{4 !^{2}} G_{b_{1} \ldots b_{4}} G_{b_{5} \ldots b_{8}} \gamma^{b_{1} \ldots b_{8}} \varepsilon \\
= & -\frac{1}{4}\left(\mathcal{R}(* 1)_{b_{1} \ldots b_{11}}-\frac{1}{6}(G \wedge * G)_{b_{1} \ldots b_{11}}\right) \gamma^{b_{1} \ldots b_{11}} \varepsilon \\
& -\frac{1}{4} \frac{1}{3}\left(\frac{1}{7 !} \nabla_{b_{1}}(* G)_{b_{2} \ldots b_{8}}+\frac{1}{2} \frac{1}{4 !^{2}} G_{b_{1} \ldots b_{4}} G_{b_{5} \ldots b_{8}}\right) \gamma^{b_{1} \ldots b_{8}} \varepsilon
\end{aligned}
$$

where in the second equality we used the Bianchi identity for the flux. As expected, we obtained the trace of Einstein-Maxwell and the $C$ gauge equation of motion - this relation is simply a result of applying a supersymmetry transformation to the equation of motion for the trace of the gravitino.

Now the action

$$
\begin{aligned}
\int \mathcal{L}_{B} & =\int \mathcal{R} * 1-\frac{1}{2} G \wedge * G-\frac{1}{6} C \wedge G \wedge G \\
& =\int \mathcal{R} * 1-\frac{1}{6} G \wedge * G-\frac{1}{3} C \wedge \mathrm{d} * G-\frac{1}{6} C \wedge G \wedge G+\text { boundary terms } \\
& =\int \mathcal{R} * 1-\frac{1}{6} G \wedge * G-\frac{1}{3} C \wedge\left(\mathrm{d} * G+\frac{1}{2} G \wedge G\right)+\text { boundary terms }
\end{aligned}
$$

so, defining a polyform $\rho$ by $\left(\tilde{\nabla}^{G} \nabla^{G} \varepsilon\right)=-\frac{1}{4} \rho \cdot \varepsilon$, we have that the bosonic Lagrangian can be written compactly in terms of the Mukai pairing ${ }^{5} \mathcal{L}_{B}=\left.\rho\right|_{11}-\left.C \wedge \rho\right|_{8}=\langle 1+C, \rho\rangle$, up to integration by parts.

\subsection{Lichnerowicz method}

This relation between the operator appearing in the supersymmetry variation of the gravitino and the bosonic action suggests we might be able to use it to build the bosonic sector of supersymmetric theories, short-cutting the usual Noether method. So let us invert the logic of the integrability calculations (3.2), and consider a general type of differential operator acting on spinors. We will then promote this Lichnerowicz equation to a necessary constraint that the operator must satisfy, rather than have it be a consequence of supersymmetry.

Let us look at what this implies more concretely. We have a metric and a 4 -form flux, and we want to build an operator that maps the supersymmetry parameter to the gravitino representation. Basic $\mathrm{SO}(10,1)$ representation theory tells us there are two ways of tensoring a 4-form with a spinor and obtain a vector-spinor (essentially corresponding to embedding in the gamma-trace or gamma-traceless part of the vector-spinor), so we

\footnotetext{
${ }^{5}$ The Mukai pairing is the top-form defined in $d$-dimensions from two polyforms $\alpha$ and $\beta$ by $\langle\alpha, \beta\rangle=$ $\sum_{p}\left(\alpha^{(p)}\right)^{T} \wedge \beta^{(d-p)}$, where $\left(\alpha^{(p)}\right)_{a_{1} \ldots a_{p}}^{T}=\alpha_{a_{p} \ldots a_{1}}^{(p)}$
} 
consider the following operators

$$
\begin{aligned}
D: S & \rightarrow T^{*} \otimes S, \\
D_{a} \varepsilon & =\nabla_{a} \varepsilon+k_{1} \gamma_{a}{ }^{b_{1} \ldots b_{4}} G_{b_{1} \ldots b_{4}} \varepsilon+k_{2} \delta_{a}{ }^{b_{1}} \gamma^{b_{2} \ldots b_{4}} G_{b_{1} \ldots b_{4}} \varepsilon, \\
\tilde{D}: T^{*} \otimes S & \rightarrow S, \\
\tilde{D}^{a} \psi_{a} & =\gamma^{a b} \nabla_{a} \psi_{b}+\tilde{k}_{1} \gamma^{a b_{1} \ldots b_{4}} G_{b_{1} \ldots b_{4}} \psi_{a}+\tilde{k}_{2} g^{a b_{1}} \gamma^{b_{2} \ldots b_{4}} G_{b_{1} \ldots b_{4}} \psi_{a},
\end{aligned}
$$

where $k_{1}, k_{2}, \tilde{k}_{1}$ and $\tilde{k}_{2}$ are left as arbitrary constants and note that a priori we may consider the 'conjugate' operator that appears in the fermionic action to also be generic.

Now we impose constraints to obtain a consistent Lichnerowicz-type relation from these operators. Clearly, if $\tilde{D}^{a} D_{a} \varepsilon$ is to define a tensor and not a differential operator, we must have that $\tilde{D}^{b}=\gamma^{a b} D_{a}$. This fixes $\tilde{k}_{1}$ and $\tilde{k}_{2}$ in terms of $k_{1}$ and $k_{2}$.

We thus have that $\tilde{D} \circ D$ is a linear map $S \rightarrow S$, and so it must be a combination of $p$-forms. Then we have the physical requirement that this tensor should contain only scalars and 3-forms (up to Hodge dualisation, and after imposing the Bianchi identity for $G$ ), corresponding to the degrees of freedom of the trace of the metric and the 3-form gauge field (this is also clearly a necessary condition for $\tilde{D} \circ D=0$ on-shell). This forces the ratio between $k_{1}$ and $k_{2}$ to be fixed, $k_{2}=-8 k_{1}$. Therefore, from these simple constraints we are left with just one free coefficient, which can be absorbed in the normalisation of the fluxes. We recover $D=\nabla^{G}$ and as we have just seen the M-theory Lichnerowicz $\tilde{D}^{a} D_{a} \varepsilon$ will give the bosonic action.

\section{Higher-derivative terms}

We will now try to apply this method to obtain the higher derivative effective action. A number of simplifications are assumed in what follows: we will discard terms which are higher order in derivatives than the order we are currently examining; we will ignore fluxes and Ricci terms, i.e. we will work only with the Riemann tensor which reduces to its Weyl tensor component; and we will also assume that at the $R^{4}$ level the action does not contain 'bare' connections, so we will not consider a term like $\nabla R \nabla R^{2}$ for example.

Even with these simplifications, the form of the possible operators remains substantially less straightforward than in the classical flux case. In particular, in moving to higher order we should expect the corrections to include derivatives of the supersymmetry parameter or the gravitino, cf. $[13,34,35]$, which means that requiring that the M-theoretic Lichnerowicz equation defines a tensor is a bit more subtle. In particular, we will find we must abandon the classical relation $\tilde{D}^{a}=\gamma^{a b} D_{a}$.

Consider, for example, a correction term corresponding to some tensor $X_{a b c d}$ which transforms in the representation $X_{a b c d} \in[0,2,0,0,0]$, that is, it has the same symmetry properties of the Weyl tensor. Note we are using the highest-weight notation of the computer algebra program LiE [38], which we used extensively throughout this work for group 
theoretic calculations. We can define the following operators

$$
\begin{aligned}
D: S & \rightarrow T^{*} \otimes S, \\
D_{a} \varepsilon & =\nabla_{a} \varepsilon+k_{1}\left(\nabla^{b} X_{a b c d}\right) \gamma^{c d} \varepsilon+k_{2} X_{a b c d} \gamma^{c d} \nabla^{b} \varepsilon, \\
\tilde{D}: T^{*} \otimes S & \rightarrow S, \\
\tilde{D}^{a} \psi_{a} & =\gamma^{a b}\left(\nabla_{a} \psi_{b}+\tilde{k}_{1}\left(\nabla^{c} X_{a c e f}\right) \gamma^{e f} \psi_{b}+\tilde{k}_{2} X_{a c e f} \gamma^{e f} \nabla^{c} \psi_{b}\right),
\end{aligned}
$$

where $k_{1}, k_{2}$ etc. parametrise higher-order corrections.

Then,

$$
\begin{aligned}
(\tilde{D} D \varepsilon)= & \gamma^{a b} \nabla_{a} \nabla_{b} \varepsilon+\left(k_{1}-\tilde{k}_{1}-k_{2}\right)\left(\nabla^{a} X_{a b c d}\right) \gamma^{c d} \nabla^{b} \varepsilon \\
& -k_{1}\left(\nabla^{a} \nabla^{b} X_{a b c d}\right) \gamma^{c d} \varepsilon-\left(\tilde{k}_{2}+k_{2}\right) X_{a b c d} \gamma^{c d} \nabla^{a} \nabla^{b} \varepsilon \\
& + \text { higher order, }
\end{aligned}
$$

so we have that, once we discard the higher order terms, tensoriality requires $k_{1}-\tilde{k}_{1}-k_{2}=0$. Note that it is crucial that the term with two covariant derivatives acting on the spinor is antisymmetrised on those $\nabla$, which is a consequence of the symmetry properties of $X_{a b c d}{ }^{6}$

Solving the coefficient constraint for $\tilde{k}_{1}$, we are left with

$$
\begin{aligned}
(\tilde{D} D \varepsilon)= & -\frac{1}{4} \mathcal{R} \varepsilon+\frac{1}{2}\left(2 k_{1}-\tilde{k}_{2}-k_{2}\right) R^{a b e}{ }_{c} X_{a b e d} \gamma^{c d} \varepsilon \\
& +\frac{1}{4}\left(\tilde{k}_{2}+k_{2}\right) R^{a b c d} X_{a b c d} \varepsilon-\frac{1}{8}\left(\tilde{k}_{2}+k_{2}\right) R^{a b}{ }_{c d} X_{a b e f} \gamma^{c d e f} \varepsilon \\
& + \text { higher order. }
\end{aligned}
$$

So, whereas we would like $\tilde{D}$ to be determined by $D$, we are left with ambiguities.

The choice we will be making from now on is to always take $\tilde{k}_{1}=0, k_{1}=k_{2}=\tilde{k}_{2}$. This means that both $\tilde{D}^{a} \nabla_{a} \varepsilon$ and $\gamma^{a b} \nabla_{a} D_{b} \varepsilon$ are separately tensorial, which can be interpreted as writing the fermionic action in terms of "supercovariant" objects [34, 35]. We thus have that $\tilde{D}$ is completely fixed given a $D$ to a certain order, and so, writing explicit spinor indices, we will consider operators in the form

$$
\begin{aligned}
D_{a} \varepsilon^{\alpha} & =\nabla_{a} \varepsilon^{\alpha}+k \nabla_{b}\left(\Theta_{a}{ }^{b} \beta^{\alpha} \varepsilon^{\beta}\right)=\nabla_{b}\left(\left(\mathbb{I}_{a}{ }^{b}{ }^{\alpha}+k \Theta_{a}{ }^{b} \beta^{\alpha}\right) \varepsilon^{\beta}\right), \\
\tilde{D}^{a} \psi_{a}{ }^{\alpha} & =\gamma^{a c}{ }^{\alpha}{ }^{\alpha}\left(\nabla_{a} \psi_{c}+k \Theta_{a}{ }^{b} \gamma^{\beta} \nabla_{b} \psi_{c}{ }^{\gamma}\right)=\gamma^{a c}{ }^{\alpha}{ }^{\alpha}\left(\mathbb{I}_{a}{ }^{b} \gamma^{\beta}+k \Theta_{a}{ }^{b} \gamma^{\beta}\right) \nabla_{b} \psi_{c}{ }^{\gamma},
\end{aligned}
$$

with $\Theta$ some object that is a function of (powers of) the Riemann curvature.

\section{1 $R^{2}$ and $R^{3}$ couplings in the effective action}

It is well known that the first corrections to the eleven-dimensional action begin at $R^{4}$. However, as a simple exercise let us check whether we can define operators that through Lichnerowicz would be compatible with an $R^{2}$ or $R^{3}$ action.

\footnotetext{
${ }^{6}$ If the tensor $X_{a b c d}$ were, say, fully symmetric, we would have extra constraints in order to ensure tensoriality. We would also not obtain extra Riemann tensors, instead we would be left with 'naked' covariant derivatives.
} 


\begin{tabular}{|ccc|}
\hline Projection of $R^{2}$ & Rep of $\mathrm{SO}(10,1)$ & Multiplicity \\
\hline$\hat{X}^{i}$ & {$[0,2,0,0,0]$} & 2 \\
$\hat{W}$ & {$[2,0,0,0,0]$} & 1 \\
$\hat{S}$ & {$[0,0,0,0,0]$} & 1 \\
\hline$\hat{T}$ & {$[0,0,0,1,0]$} & 1 \\
\hline
\end{tabular}

Table 1. Valid embeddings of $\otimes^{2} R$ in $\delta \psi$.

Counting derivatives, we see that in order to obtain an $R^{2}$ correction to the action, there is only one type of term we can add to the operators, namely

$$
\begin{aligned}
D_{a} \varepsilon & =\nabla_{a} \varepsilon+k \nabla^{b}\left(R_{a b c d} \gamma^{c d} \varepsilon\right), \\
\tilde{D}^{a} \psi_{a} & =\gamma^{a b} \nabla_{a} \psi_{b}+k R_{a b c d} \gamma^{c d} \nabla^{b} \psi_{a} .
\end{aligned}
$$

However, it is clear that $\tilde{D}^{a} D_{a} \varepsilon$ will have a 4 -form, $k R_{a_{1} a_{2} b_{1} b_{2}} R_{a_{3} a_{4} b_{1} b_{2}} \gamma^{a_{1} a_{2} a_{3} a_{4}} \varepsilon$, so satisfying the constraint that $\tilde{D} D \varepsilon$ should contain only scalars and 3 -forms forces $k=0$. We conclude no corrections are admissible at this order.

For $R^{3}$ there are more possibilities. By derivative counting we have that the extra pieces in $D$ must be of the type $\nabla R^{2}$. So we look at the tensor decomposition of $\otimes^{2} R$ and find that the families of terms that can be considered in the operators are the ones listed in table 1.

Explicitly, these decompositions are given by

$$
\begin{aligned}
\hat{X}_{a_{1} a_{2} c_{1} c_{2}}^{1}= & \frac{1}{2} R_{a_{1} a_{2} b_{1} b_{2}} R_{c_{1} c_{2} b_{1} b_{2}}+\frac{1}{2} R_{a_{1} c_{2} b_{1} b_{2}} R_{c_{1} a_{2} b_{1} b_{2}} \\
& -\frac{1}{3} g_{a 2 c 2} R_{a_{1} d_{1} b_{1} b_{2}} R_{c_{1} d_{1} b_{1} b_{2}}+\frac{1}{60} g_{a 1 c 1} g_{a 2 c 2} R_{d_{1} d_{2} b_{1} b_{2}} R_{d_{1} d_{2} b_{1} b_{2}}, \\
\hat{X}_{a_{1} a_{2} c_{1} c_{2}}^{2}= & \frac{1}{2} R_{a_{1} b_{1} c_{1} b_{2}} R_{a_{2} b_{1} c_{2} b_{2}}+\frac{1}{2} R_{c_{1} b_{1} a_{1} b_{2}} R_{a_{2} b_{1} c_{2} b_{2}} \\
& +\frac{1}{6} g_{a 2 c 2} R_{a_{1} d_{1} b_{1} b_{2}} R_{c_{1} d_{1} b_{1} b_{2}}-\frac{1}{120} g_{a 1 c 1} g_{a 2 c 2} R_{d_{1} d_{2} b_{1} b_{2}} R_{d_{1} d_{2} b_{1} b_{2}}, \\
\hat{W}_{a_{1} c_{1}}= & R_{a_{1} d_{1} b_{1} b_{2}} R_{c_{1} d_{1} b_{1} b_{2}}-\frac{1}{11} g_{a_{1} c_{1}} R_{d_{1} a_{2} b_{1} b_{2}} R_{d_{1} a_{2} b_{1} b_{2}}, \\
\hat{S}= & R_{a_{1} a_{2} b_{1} b_{2}} R_{a_{1} a_{2} b_{1} b_{2}}, \\
\hat{T}_{a_{1} a_{2} a_{3} a_{4}}= & R_{a_{1} a_{2} b_{1} b_{2}} R_{a_{3} a_{4} b_{1} b_{2}},
\end{aligned}
$$

where same-letter free indices are assumed to be antisymmetrised. However, the $\hat{W}$ and $\hat{S}$ do not actually contribute to the Lichnerowicz (they are projected out) so we will not consider them as they cannot possibly source corrections to the action.

There is now a new potential ambiguity in how to construct the supersymmetry operators, as there are several different ways of embedding the $\hat{T}$ term in $\delta \psi$. We will discuss this in more detail in the $R^{4}$ section, but some of these can be fixed by consistency conditions from the Lichnerowicz equation, and the rest by requiring that the $\hat{T}$ embed strictly into the traceless-vector-spinor in the conjugate operator $\tilde{D}$, with the trace taken from the 
right so that the operator $\tilde{D}$ is now the one that appears in the fermionic coupling of the gravitino trace $\int \tilde{\psi} \tilde{D} \psi$. This will imply that $\tilde{D}^{a} \gamma_{a}=\nabla^{a} \gamma_{a}$, as the $\hat{X}^{i}$ likewise only embed in the traceless part. This also ensures that $\tilde{\not D}=\tilde{\not D}^{\dagger}$.

We end up with

$$
\begin{aligned}
D_{a} \varepsilon= & \nabla_{a} \varepsilon+\sum_{i=1}^{2} \hat{x}_{i} \gamma^{c d} \nabla^{b}\left(\hat{X}_{a b c d}^{i} \varepsilon\right) \\
+ & \hat{t}\left(\gamma^{b_{1} b_{2}} \nabla^{b_{3}}\left(\hat{T}_{a b_{1} b_{2} b_{3}} \varepsilon\right)+\frac{1}{14} \gamma^{b_{1} \ldots b_{4}} \nabla_{a}\left(\hat{T}_{b_{1} \ldots b_{4}} \varepsilon\right)+\frac{1}{14} \gamma^{b_{1} \ldots b_{4}} \nabla_{b_{1}}\left(\hat{T}_{a b_{2} \ldots b_{4}} \varepsilon\right)\right. \\
& \left.+\frac{1}{7} \gamma_{a}{ }^{b_{2} b_{3} b_{4}} \nabla^{b_{1}}\left(\hat{T}_{b_{1} \ldots b_{4}} \varepsilon\right)+\frac{1}{84} \gamma_{a}^{b_{1} \ldots b_{5}} \nabla_{b_{5}}\left(\hat{T}_{b_{1} \ldots b_{4}} \varepsilon\right)\right)
\end{aligned}
$$

and

$$
\begin{aligned}
\tilde{D}^{a} \psi_{a}= & \gamma^{a b} \nabla_{a} \psi_{b}+\sum_{i=1}^{2} \hat{x}_{i} \gamma^{c d} \hat{X}_{a b c d}^{i} \nabla^{b} \psi^{a} \\
& +\hat{t}\left(\frac{5}{2} \gamma^{c_{1} c_{2}} \hat{T}_{a b c_{1} c_{2}} \nabla^{a} \psi^{b}+\frac{5}{14} \gamma^{a c_{2} c_{3} c_{4}} \hat{T}_{c_{1} \ldots c_{4}}\left(\nabla_{a} \psi^{c_{1}}-\nabla^{c_{1}} \psi_{a}\right)\right. \\
& \left.-\frac{5}{84} \gamma^{a b c_{1} \ldots c_{4}} \hat{T}_{c_{1} \ldots c_{4}} \nabla_{a} \psi_{b}\right),
\end{aligned}
$$

so we have a priori three free coefficients, $\hat{x}_{1}, \hat{x}_{2}, \hat{t}$. If we compute $\tilde{D}^{a} D_{a} \varepsilon$ we find that in addition to scalars in $R^{3}$, the Lichnerowicz also contains 4 -forms, which must be cancelled. There are exactly three possible independent $R^{3} 4$-forms, all of which appear in computation, and eliminating them forces precisely that all $\hat{x}_{i}=\hat{t}=0$. We conclude that no $R^{3}$ corrections are admissible.

\section{$4.2 \quad R^{4}$ couplings}

Finally, we arrive at corrections to the supersymmetry operators that may lead to $R^{4}$ corrections to the action via the Lichnerowicz procedure. We will need to add $\nabla R^{3}$ terms to $\delta \psi$, for which there are several possibilities depending on how we build the $R^{3}$ factor, as listed in table 2.

We find these families of terms by decomposing into irreducible representations the product of three Weyl tensors, i.e. if we denote the Weyl curvature representation $[0,2,0,0,0]$ by $W$, we consider the symmetrised cubic tensor product $S^{3} W$. Then we restrict to those which, when combined with a $\nabla_{a}$ and a spinor $\varepsilon$, can contain a vector spinor $\delta \psi_{a}$. In other words, we look for the irreducible representations in the intersection $S^{3} W \cap(V \otimes S)^{*} \otimes(V \otimes S)$. We then verify these projections by explicit construction they are given in appendix A.1.

Note that in some cases there are multiple ways of embedding these terms into a vector spinor. For example, consider the three $T^{i}$, each of which is a 4 -form. There are five inequivalent ways of combining a 4 -form together with $\nabla_{a}$ and $\varepsilon$ to obtain a valid $\delta \psi_{a}$. These roughly correspond to using either a $\gamma^{(2)}$, a $\gamma^{(4)}$ or a $\gamma^{(6)}$ to "soak up" the indices, and then further distinguishing in the first two whether the overall free index is 


\begin{tabular}{|ccc|}
\hline Projection of $R^{3}$ & Rep of $\mathrm{SO}(10,1)$ & Multiplicity \\
\hline$X^{i}$ & {$[0,2,0,0,0]$} & 8 \\
$W^{i}$ & {$[2,0,0,0,0]$} & 3 \\
$S^{i}$ & {$[0,0,0,0,0]$} & 2 \\
\hline$Y^{i}$ & {$[0,1,0,0,2]$} & 2 \\
$V^{i}$ & {$[1,0,0,0,2]$} & 2 \\
$T^{i}$ & {$[0,0,0,1,0]$} & 3 \\
\hline$Z^{i}$ & {$[0,1,0,1,0]$} & 3 \\
$U^{i}$ & {$[1,0,1,0,0]$} & 3 \\
\hline$L^{i}$ & {$[2,1,0,0,0]$} & 3 \\
$M^{i}$ & {$[2,0,0,1,0]$} & 6 \\
\hline
\end{tabular}

Table 2. Valid embeddings of $\otimes^{3} R$ in $\delta \psi$. In each case, the index $i$ runs over the corresponding multiplicity.

symmetrised or antisymmetrised with the index on $\nabla_{a}$. So a priori, the $T^{i}$ would appear to contribute 15 undetermined coefficients to our operators.

There are, however, some constraints from the M-theoretic Lichnerowicz that can be immediately applied to reduce the number of possibilities. We already established we only want antisymmetrised products of $\nabla$ appearing in $\tilde{D}^{a} D_{a} \varepsilon$, so that they will be converted into a Riemann tensor. Symmetrised $\nabla$ would lead to 'bare' connections in the action and could even spoil the tensoriality of the Lichnerowicz. This constraint eliminates the last two terms in the table, $L^{i}$ and $M^{i}$, and further reduces the admissible embeddings of some of the other terms. For instance, the $T^{i}$ can now each only be embedded in three different ways, for a total of 9 free coefficients.

Finally, we impose the condition that only the traceless part of the $\tilde{D}$ operator gets modified which, as mentioned in the previous subsection, is sufficient to ensure that $\tilde{D}=$ $\tilde{D}^{\dagger}$. It turns out that this is enough to fix these ambiguities of embedding, and the total number of remaining free coefficients matches precisely the multiplicity of projections of $R^{3}$.

Now, the representation theory also tells us what type of $p$-forms to expect in the Lichnerowicz. Since we have ensured that this expression will be, schematically, $R^{3}[\nabla, \nabla] \varepsilon=R^{4} \varepsilon$, we need to look at the $p$-forms in the symmetric tensor product of four Weyl curvatures and see how each of the remaining terms in table might contribute to them. We summarise the result in table 3 .

We immediately observe that, as previously remarked, terms of type $W^{i}$ and $S^{i}$ are projected out in the Lichnerowicz computation, and so their presence (or lack thereof) in the supersymmetry operator cannot be constrained in this manner. We also see that we need to have $X^{i}$ type terms if we hope to find corrections to the scalars, and $Y^{i}$ for corrections to the 3 -form. These are the only objects that may contribute to a corrected action. However, they will also give rise to 4 -forms which cannot be cancelled solely by picking appropriate 


\begin{tabular}{|c|cccccc|}
\hline$p$-form : & 0 & 1 & 2 & 3 & 4 & 5 \\
\hline$R^{4}$ multiplicity: & 7 & 0 & 1 & 2 & 17 & 0 \\
\hline$X^{i} \otimes R$ & $\bullet$ & - & $\bullet$ & - & $\bullet$ & - \\
$W^{i} \otimes R$ & - & - & - & - & - & - \\
$S^{i} \otimes R$ & - & - & - & - & - & - \\
\hline$Y^{i} \otimes R$ & - & - & - & $\bullet$ & $\bullet$ & - \\
$V^{i} \otimes R$ & - & - & - & - & $\bullet$ & - \\
$T^{i} \otimes R$ & - & - & - & - & $\bullet$ & - \\
\hline$Z^{i} \otimes R$ & - & - & $\bullet$ & - & $\bullet$ & - \\
$U^{i} \otimes R$ & - & - & $\bullet$ & - & $\bullet$ & - \\
\hline
\end{tabular}

Table 3. Potential contributions of the terms in table 2 to different $p$-forms in the M-theoretic Lichnerowicz.

coefficients $x_{i}$ and $y_{i}$ for each of them. Instead, we find by explicit computation that we must, at a minimum, also add terms of type $V^{i}$ and $T^{i}$, as we will now describe.

\subsubsection{Minimal solution}

Given these ingredients, we build:

$$
\begin{aligned}
D: S \rightarrow & T^{*} \otimes S \\
D_{a} \varepsilon= & \nabla_{a} \varepsilon+\sum_{i=1}^{8} x_{i} \gamma^{c d} \nabla^{b}\left(X_{a b c d}^{i} \varepsilon\right)+\sum_{i=1}^{2} y_{i} \gamma^{c_{1} \ldots c_{6}} \nabla^{b}\left(Y_{a b c_{1} \ldots c_{6}}^{i} \varepsilon\right) \\
& +\sum_{i=1}^{3} t_{i}\left(\gamma^{b_{1} b_{2}} \nabla^{b_{3}}\left(T_{a b_{1} b_{2} b_{3}}^{i} \varepsilon\right)+\frac{1}{14} \gamma^{b_{1} \ldots b_{4}} \nabla_{a}\left(T_{b_{1} \ldots b_{4}}^{i} \varepsilon\right)+\frac{1}{14} \gamma^{b_{1} \ldots b_{4}} \nabla_{b_{1}}\left(T_{a b_{2} \ldots b_{4}}^{i} \varepsilon\right)\right. \\
& \left.\quad+\frac{1}{7} \gamma_{a}^{b_{2} b_{3} b_{4}} \nabla^{b_{1}}\left(T_{b_{1} \ldots b_{4}}^{i} \varepsilon\right)+\frac{1}{84} \gamma_{a}^{b_{1} \ldots b_{5}} \nabla_{b_{5}}\left(T_{b_{1} \ldots b_{4}}^{i} \varepsilon\right)\right) \\
& +\sum_{i=1}^{2} v_{i}\left(\frac{1}{4} \gamma^{b_{1} \ldots b_{4}} \nabla^{b_{5}}\left(V_{a b_{1} \ldots b_{5}}^{i} \varepsilon-V_{b_{5} a b_{1} \ldots b_{4}}^{i} \varepsilon\right)+\frac{1}{25} \gamma_{a}^{b_{1} \ldots b_{5}} \nabla^{c}\left(V_{c b_{1} \ldots b_{5}}^{i} \varepsilon\right)\right. \\
& \left.\quad+\frac{1}{25} \gamma^{b_{1} \ldots b_{6}} \nabla_{b_{6}}\left(V_{a b_{1} \ldots b_{5}}^{i} \varepsilon\right)\right) .
\end{aligned}
$$

As mentioned, these combinations ensure that Lichnerowicz will not contain symmetrised $\nabla$. In other words, we have that $\tilde{D} D \varepsilon=\sum_{n}\left(R^{4}\right)_{a_{1} \ldots a_{n}} \gamma^{a_{1} \ldots a_{n}} \varepsilon$ with no 'bare' connections 
left. This should be clear once we look at the conjugate $\tilde{D}$ operator:

$$
\begin{aligned}
& \tilde{D}: T^{*} \otimes S \rightarrow \\
& \tilde{D}^{a} \psi_{a}=\gamma^{a b} \nabla_{a} \psi_{b}+\sum_{i=1}^{8} x_{i} \gamma^{c d} X_{a b c d}^{i} \nabla^{b} \psi^{a}+\sum_{i=1}^{2} y_{i} \gamma^{c_{1} \ldots c_{6}} Y_{a b c_{1} \ldots c_{6}}^{i} \nabla^{b} \psi^{a} \\
&+\sum_{i=1}^{3} t_{i}\left(\frac{5}{2} \gamma^{c_{1} c_{2}} T_{a b c_{1} c_{2}}^{i} \nabla^{a} \psi^{b}+\frac{5}{14} \gamma^{a c_{2} c_{3} c_{4}} T_{c_{1} \ldots c_{4}}^{i}\left(\nabla_{a} \psi^{c_{1}}-\nabla^{c_{1}} \psi_{a}\right)-\frac{5}{84} \gamma^{a b c_{1} \ldots c_{4}} T_{c_{1} \ldots c_{4}}^{i} \nabla_{a} \psi_{b}\right) \\
&+\sum_{i=1}^{2} v_{i}\left(\gamma^{a c_{3} c_{4} c_{5}} V_{a c_{1} \ldots c_{5}}^{i} \nabla^{c_{1}} \psi^{c_{2}}+\frac{1}{25} \gamma^{b c_{1} \ldots c_{5}} V_{a c_{1} \ldots c_{5}}^{i}\left(\nabla_{b} \psi^{a}-\nabla^{a} \psi_{b}\right)\right)
\end{aligned}
$$

Note that $\tilde{D}^{a} \gamma_{a}=\not \varnothing$ as required.

Now we need to fix the coefficients such that only scalars and 3-forms survive. We used the computer program Cadabra [20,21] to solve this algebra problem (and also performed some double-checks on Mathematica). The single possible $R^{4} 2$-form vanishes identically and eliminating the 4 -forms is solved by:

$$
\begin{aligned}
& x_{1}=-y_{2}, \quad x_{7}=16 y_{2}, \quad t_{2}=\frac{2}{45}\left(72 y_{2}-x_{5}\right), \\
& x_{2}=16 y_{2}, \quad x_{8}=16 y_{2}, \quad t_{3}=-\frac{4}{45}\left(72 y_{2}-x_{5}\right) \text {, } \\
& x_{3}=-2 y_{2}, \quad y_{1}=-\frac{1}{4} y_{2}, \quad v_{2}=\frac{5}{8}\left(-24 y_{2}+5 x_{5}\right), \\
& x_{4}=-16 y_{2}, \quad t_{1}=0, \\
& x_{6}=-4 x_{5}, \quad v_{1}=0,
\end{aligned}
$$

with $y_{2}, x_{5}$ free. We have therefore found two solutions for our operators at $R^{4}$ such that the Lichnerowicz contains only scalars and 3-forms (or rather, what results naturally from the computation are their Hodge-dual 8-forms)

$$
\tilde{D}^{a} D_{a} \varepsilon=(\text { scalar }) \varepsilon+(8 \text {-form })_{a_{1} \ldots a_{8}} \gamma^{a_{1} \ldots a_{8}} \varepsilon
$$

Note that in the notation of $[27,34,35]$, we have a basis for the seven different $R^{4}$ scalars

$$
\begin{array}{lll}
R \cdot X^{1}=R_{44}, & R \cdot X^{2}=R_{45}, & R \cdot X^{3}=R_{43}, \\
R \cdot X^{4}=R_{41}, & R \cdot X^{5}=R_{46}, & R \cdot X^{6}=A_{7}, \\
R \cdot X^{7}=-R_{42}-\frac{1}{4} R_{46}+A_{7}, & R \cdot X^{8}=-R_{42}+\frac{1}{4} R_{46}+A_{7}, &
\end{array}
$$

where $R \cdot X^{i}=R^{a b c d} X_{a b c d}^{i}$ and so the scalar component of the M-theory Lichnerowicz can be written as (below we write the Hilbert-Einstein term for convenience; other terms 
containing the Ricci tensor or scalar are dropped)

$$
\begin{aligned}
\left(\left.\tilde{D}^{a} D_{a} \varepsilon\right|_{\text {scalar }}\right)= & -\frac{1}{4} \mathcal{R}+\frac{1}{2} x_{5}\left(R_{46}-4 A_{7}\right) \\
& +\frac{1}{2} y_{2}\left(-16 R_{41}-32 R_{42}-2 R_{43}-R_{44}+16 R_{45}+32 A_{7}\right) \\
= & -\frac{1}{4} \mathcal{R}-\frac{1}{2} \frac{1}{192} x_{5}\left(-192 R_{46}+768 A_{7}\right) \\
& -\frac{1}{2} \frac{1}{12} y_{2}\left(192 R_{41}+384 R_{42}+24 R_{43}+12 R_{44}-192 R_{45}-384 A_{7}\right) \\
= & -\frac{1}{4} \mathcal{R} \\
& -\frac{1}{4} \frac{1}{96} x_{5}\left(t_{8} t_{8} R^{4}-\frac{1}{4} E_{8}\right)-\frac{1}{4} \frac{1}{12} y_{2}\left(t_{8} t_{8} R^{4}+\frac{1}{4} E_{8}\right),
\end{aligned}
$$

while the 8-form is:

$$
\begin{aligned}
\left(\left.\tilde{D}^{a} D_{a} \varepsilon\right|_{8 \text {-form }}\right)_{a_{1} \ldots a_{8}}= & -\frac{1}{4} y_{2}\left(-\frac{1}{4} R \cdot Y^{1}+R \cdot Y^{2}\right)_{a_{1} \ldots a_{8}} \\
=-\frac{1}{4} y_{2}( & -\frac{1}{4} R_{a_{1} a_{2} b_{1} b_{2}} R_{a_{3} a_{4} b_{1} b_{2}} R_{a_{5} a_{6} c_{1} c_{2}} R_{a_{7} a_{8} c_{1} c_{2}} \\
& \left.\quad+R_{a_{1} a_{2} b_{1} c_{1}} R_{a_{3} a_{4} c_{1} c_{2}} R_{a_{5} a_{6} c_{2} b_{2}} R_{a_{7} a_{8} b_{2} b_{1}}\right)
\end{aligned}
$$

so $\frac{1}{16} R \cdot Y^{1}=\operatorname{tr} R^{2} \wedge \operatorname{tr} R^{2}$ and $\frac{1}{16} R \cdot Y^{2}=\operatorname{tr} R^{4}$. We thus recognise the $y_{2} \neq 0$ solution as corresponding to the invariant $\left(t_{8} t_{8} R^{4}+\frac{1}{4} E_{8}\right)$ and $x_{5} \neq 0$ to $\left(t_{8} t_{8} R^{4}-\frac{1}{4} E_{8}\right)$. To get the correct eleven-dimensional action we thus need to take $x_{5}=0$ and $y_{2}$ will be fixed by the normalisation of the higher derivative terms. The existence of this freedom of choice is maybe not unexpected, as three different superinvariants had already been identified previously [39]. Comparing with [34,35] we have, in the notation of that paper, that the $y_{2}$ terms give the $I_{X}+\frac{1}{8} I_{Z}$ invariant, while the $x_{5}$ terms give $I_{X}-\frac{1}{8} I_{Z}$.

In appendix $B$ we discuss other possible solutions to this Lichnerowicz system.

\section{Application: seven-dimensional internal spaces}

Two out of the three higher-derivative structures allowed by supersymmetry involve a complete antisymmetrisation of eight indices. Hence, for our computation, seven is a sort of critical dimension, i.e. the highest where only a single structure survives. So, in addition to the natural desire of learning more about M-theory compactifications on seven-dimensional internal spaces, assuming the eleven-dimensional space breaks as $\mathcal{M}_{11}=\mathcal{M}_{4} \times \mathcal{M}_{7}$ and focusing on the $\mathcal{M}_{7}$ component provides a good setting to make further considerations on our construction. ${ }^{7}$ We then have that the two eleven-dimensional scalars $y_{2}$ and $x_{5}$ will coincide $\left(t_{8} t_{8} R^{4}+\frac{1}{4} E_{8}\right)=\left(t_{8} t_{8} R^{4}-\frac{1}{4} E_{8}\right)=t_{8} t_{8} R^{4}$ when fully restricted to $\mathcal{M}_{7}$, and even though the

\footnotetext{
${ }^{7}$ Of course, a calculation on $\mathcal{M}_{7}$ can be considered on its own, with the view of deriving higher-derivative corrections in seven-dimensional effective theories. We mostly concentrate on supersymmetry on the internal seven-manifold.
} 
$y_{2}$ is the correct physical solution in eleven dimensions, in seven dimensions we may just as well work with the simpler $x_{5}$ when writing a fully restricted operator $D$. In terms of $\mathrm{SO}(7)$ representations using the projectors of the cubic powers of $R_{m n p q}$ in appendix A.2, this is

$$
\begin{aligned}
D_{m} \varepsilon= & \nabla_{m} \varepsilon+x_{5} \gamma^{p q} \nabla^{n}\left(\left(\check{X}_{m n p q}^{5}-4 \check{X}_{m n p q}^{6}\right) \varepsilon\right)+\frac{2}{315} x_{5} \gamma_{m}{ }^{n} \nabla_{n}\left(\left(\check{S}^{1}-2 \check{S}^{2}\right) \varepsilon\right) \\
& +\frac{8}{45} x_{5} \gamma^{n p} \nabla_{n}\left(\left(-\check{W}_{m p}^{1}+2 \check{W}_{m p}^{2}+\check{W}_{m p}^{3}\right) \varepsilon\right)-\frac{8}{45} x_{5} \gamma_{m}{ }^{n} \nabla^{p}\left(\left(-\check{W}_{p n}^{1}+2 \check{W}_{p n}^{2}+\check{W}_{p n}^{3}\right) \varepsilon\right) \\
+ & x_{5}\left(-\frac{2}{45} \gamma^{n_{1} n_{2}} \nabla^{n_{3}}\left(\check{T}_{m n_{1} n_{2} n_{3}}^{2} \varepsilon\right)-\frac{1}{315} \gamma^{n_{1} \ldots n_{4}} \nabla_{m}\left(\check{T}_{n_{1} \ldots n_{4}}^{2} \varepsilon\right)-\frac{379}{1260} \gamma^{n_{1} \ldots n_{4}} \nabla_{n_{1}}\left(\check{T}_{m n_{2} \ldots n_{4}}^{2} \varepsilon\right)\right. \\
& \left.+\frac{367}{1260} \gamma_{m}{ }^{n_{2} n_{3} n_{4}} \nabla^{n_{1}}\left(\check{T}_{n_{1} \ldots n_{4}}^{2} \varepsilon\right)-\frac{113}{945} \gamma_{m}^{n_{1} \ldots n_{5}} \nabla_{n_{5}}\left(\check{T}_{n_{1} \ldots n_{4}}^{2} \varepsilon\right)\right) \\
+ & x_{5}\left(\frac{4}{45} \gamma^{n_{1} n_{2}} \nabla^{n_{3}}\left(\check{T}_{m n_{1} n_{2} n_{3}}^{3} \varepsilon\right)+\frac{2}{315} \gamma^{n_{1} \ldots n_{4}} \nabla_{m}\left(\check{T}_{n_{1} \ldots n_{4}}^{3} \varepsilon\right)+\frac{11}{45} \gamma^{n_{1} \ldots n_{4}} \nabla_{n_{1}}\left(\check{T}_{m n_{2} \ldots n_{4}}^{3} \varepsilon\right)\right. \\
& \left.-\frac{71}{315} \gamma_{m}^{n_{2} n_{3} n_{4}} \nabla^{n_{1}}\left(\check{T}_{n_{1} \ldots n_{4}}^{3} \varepsilon\right)+\frac{13}{135} \gamma_{m}{ }^{n_{1} \ldots n_{5}} \nabla_{n_{5}}\left(\check{T}_{n_{1} \ldots n_{4}}^{3} \varepsilon\right)\right) \\
+\frac{25}{8} x_{5}\left(\frac{1}{4} \gamma^{n_{1} \ldots n_{4}} \nabla^{n_{5}}\left(\check{V}_{m n_{1} n_{2} n_{3} n_{4} n_{5}}^{2} \varepsilon-\check{V}_{\left.n_{5} m n_{1} n_{2} n_{3} n_{4} \varepsilon\right)}^{2} \varepsilon\right)\right. & \left.+\frac{1}{25} \gamma_{m}^{n_{1} \ldots n_{5}} \nabla^{p}\left(\check{V}_{p n_{1} n_{2} n_{3} n_{4} n_{5}}^{2} \varepsilon\right)+\frac{1}{25} \gamma^{n_{1} \ldots n_{6}} \nabla_{n_{6}}\left(\check{V}_{m n_{1} n_{2} n_{3} n_{4} n_{5}}^{2} \varepsilon\right)\right)
\end{aligned}
$$

where $m, n, \ldots$ are internal seven-dimensional indices and now $\nabla$ is the Levi-Civita of the internal manifold and $R_{m n p q}$ its (Weyl) curvature.

The full decomposition, keeping dependence on both internal and external contributions, will be much more involved and we will not perform it here. However, we can try to capture some aspects by considering a restricted Lichnerowicz formula in the sevendimensional space, expanded in order of internal derivatives. As we will soon see, this seven-dimensional Lichnerowicz formula has corrections starting from three-derivatives on the operators and will turn out to reveal some interesting new structures. In order to relate it to its eleven-dimensional counterpart, one needs to think of the coefficients in these operators as being made of Riemann curvatures in the external (four-dimensional) spacetime. ${ }^{8}$

\subsection{M-theory Lichnerowicz for $\mathrm{SO}(7)$}

In performing the M-theory Lichnerowicz in seven dimensions, we may immediately expect one difference. The eleven-dimensional Lichnerowicz was picking zero- and eight-forms in the expansion of $\rho$. In seven-dimensions, one is instead interested in zero- and four-form parts and the action, up to integration by parts, is given by:

$$
\mathcal{L}_{B}=\left.\rho\right|_{7}-\left.C \wedge \rho\right|_{4}=\langle 1+C, \rho\rangle .
$$

\footnotetext{
${ }^{8}$ In order to make proper contact with the eleven-dimensional calculation, one should strictly start with a zero-derivative term $D_{m} \varepsilon=\nabla_{m} \varepsilon+\Lambda \gamma_{m} \varepsilon$, corresponding to taking all the $R^{4}$ couplings in the external four-dimensional space, and which would lead to a constant piece in the internal action $\tilde{D}^{m} D_{m} \varepsilon=$ $-\frac{1}{4}\left(\mathcal{R}+168 \Lambda^{2}\right) \varepsilon$. To keep the calculation similar to the eleven-dimensional one, we will ignore this term.
} 


\subsubsection{Three-derivative terms in the operators}

In section 4.1 we used a very simple argument to rule out any four-derivative contributions to the eleven-dimensional action - they would inevitably contribute an unphysical 4-form to the M-theory Lichnerowicz. However, in seven dimensions a 4-form is Hodge-dual to a 3 -form and thus contributes to the flux equation of motion. Therefore, we will allow the operators

$$
\begin{aligned}
D_{m} \varepsilon & =\nabla_{m} \varepsilon+k \nabla^{n}\left(R_{m n p q} \gamma^{p q} \varepsilon\right), \\
\tilde{D}^{m} \psi_{m} & =\gamma^{m n} \nabla_{m} \psi_{n}+k R_{m n p q} \gamma^{p q} \nabla^{n} \psi_{m} .
\end{aligned}
$$

These operators are not in contradiction with the eleven-dimensional construction - in fact, they should be expected. Consider the $X^{1}$ term in the solution (4.9). Explicitly $\nabla^{b} X_{a b c d}^{1}=\nabla^{b} R_{a b c d}^{11 \mathrm{~d}}\left(R^{11 \mathrm{~d}}\right)^{2}$ and one way of decomposing this is as $\nabla^{n} R_{m n p q}^{7 \mathrm{~d}}\left(R^{4 \mathrm{~d}}\right)^{2} \mapsto$ $k \nabla^{n} R_{m n p q}^{\mathrm{7d}}$. So consistency with our eleven-dimensional solution implies that $k$ is of at least quartic order in external derivatives.

The seven-dimensional Lichnerowicz now yields

$$
\tilde{D}^{m} D_{m} \varepsilon=-\frac{1}{4} \mathcal{R} \varepsilon+\frac{1}{2} k R_{m n p q} R^{m n p q} \varepsilon-\frac{1}{4} k R_{m n p q} R_{r s}{ }^{p q} \gamma^{m n r s} \varepsilon+\text { higher order. }
$$

In the effective theory the interpretation is immediate: using (5.2) we obtain the $C_{3} \wedge\left(\operatorname{tr} R^{2}\right)$ term of the theory with 16 supercharges [29] together with its (Riemann) ${ }^{2}$ completion. As for the internal supersymmetry, we may already note a major limitation of our approach. Since we are effectively integrating out the four-dimensional action, we have lost the ability to distinguish a four-dimensional scalar from a top-form. Indeed it is not hard to see that $X_{a b c d}^{1}$ is not the only source of terms $\sim k$ in (5.3). Such a term may also originate from $Y_{a_{1} a_{2} b_{1} \ldots b_{6}}^{1}$. From other side, one may already guess that like in the seven-dimensional effective theory, these will yield internal top-forms quartic in derivatives. We shall return to these in subsection 5.3.

Before turning to the discussion of contributions with a higher number of derivatives, we should remark that this interpretation results in an extra physical constraint, which is important for reducing the number of further terms. As mentioned in section 2.1, $X_{8}$ is related to M5/NS5 anomalies and string-theoretically it is one-loop and does not receive any higher loop contributions. On the other hand, the next set of corrections to the internal covariant derivatives are, from the eleven-dimensional point of view, reductions of terms which are (at least) thirteen derivatives, i.e. the types of corrections that may contribute to $R^{7}$ (and higher) couplings. These come from string two (and higher) loops, and hence should not affect $X_{8}$. This means that there can be no further higher-derivative 4 -forms in seven dimensions, as these would correct $X_{8}$ when lifted to eleven dimensions. So even if there exist six- (or higher) derivative four-form modifications to (5.4) consistent with the Lichnerowicz method, we should not allow such contributions. This in turn implies that the constant $k$ is exactly of quartic order in external derivatives.

\subsubsection{Five-derivative terms}

Furthermore, the reasoning we used in section 4.1 to rule out $\nabla R^{2}$ terms in the supersymmetry operators in eleven dimensions remains valid in seven. These necessarily add 4 -forms 
to the Lichnerowicz which we have just argued cannot be allowed. However, we are not done at this order. Since we have changed the supersymmetry operators at a lower order, we need to check whether the Lichnerowicz remains consistent at the five-derivative level - or put in another way, whether the supersymmetry algebra still closes to this order. It does not:

$$
\begin{aligned}
\tilde{D}^{m} D_{m} \varepsilon= & -\frac{1}{4} \mathcal{R} \varepsilon+\frac{1}{2} k R_{m n p q} R^{m n p q} \varepsilon-\frac{1}{4} k R_{m n p q} R_{r s}{ }^{p q} \gamma^{m n r s} \varepsilon \\
& +k^{2} R_{m n p q} \gamma^{p q} \nabla^{n} \nabla^{r}\left(R_{m r s t} \gamma^{s t} \varepsilon\right) .
\end{aligned}
$$

The last term of the Lichnerowicz does not define a tensor. We must therefore introduce a new correction piece to our operators, which will precisely cancel this last term

$$
\begin{aligned}
D_{m} \varepsilon= & \nabla_{m} \varepsilon+k \nabla^{n}\left(R_{m n p q} \gamma^{p q} \varepsilon\right) \\
& +4 k^{2} \frac{1}{6}\left(\gamma_{m n}-5 g_{m n}\right) \nabla_{p}\left(A^{n p}{ }_{q r} \gamma^{q r} \varepsilon\right), \\
\tilde{D}^{m} \psi_{m}= & \gamma^{m n} \nabla_{m} \psi_{n}+k R_{m n p q} \gamma^{p q} \nabla^{n} \psi^{m} \\
& -4 k^{2} \tilde{A}_{p q}^{m n} \gamma^{p q} \nabla_{n} \psi_{m},
\end{aligned}
$$

with

$$
\begin{aligned}
& A_{p q}^{m n} \gamma^{p q} \varepsilon=\nabla^{[m} \nabla_{p}\left(R^{n] p}{ }_{q r} \gamma^{q r} \varepsilon\right), \\
& \tilde{A}^{m n}{ }_{p q} \gamma^{p q} \varepsilon=R^{p[m}{ }_{q r} \nabla_{p} \nabla^{n]} \gamma^{q r} \varepsilon .
\end{aligned}
$$

Note that in seven dimensions, $\gamma^{m n}\left(\gamma_{n p}-5 g_{n p}\right)=6 \delta_{p}^{m}$. These new terms are not of the form $\nabla R^{n}$ which we had considered thus far, they include higher derivatives of the spinor paramenter (also seen in, for example, [14]), and clearly do not appear in the eleven-dimensional solution. Their origin is clear, however, as they come multiplied by $k^{2}$, a factor which is of order $\partial^{8}$ in external derivatives. In total, we recognise that these new terms are of the same order as $R^{7}$ corrections in eleven dimensions. If we had simply restricted ourselves to reducing the $R^{4}$ solution we would never have found these terms, but they are natural, indeed crucial, from the point of view of the internal seven-dimensional supersymmetry.

\subsubsection{Seven-derivative terms}

Of course, moving to the next order once again breaks the Lichnerowicz, and we have to introduce new corrections, proportional to $k^{3}$, and which must descend from elevendimensional terms of order $R^{10}$. We find

$$
\begin{aligned}
D_{m} \varepsilon= & \nabla_{m} \varepsilon+k \nabla^{n}\left(R_{m n p q} \gamma^{p q} \varepsilon\right) \\
& +4 k^{2} \frac{1}{6}\left(\gamma_{m n}-5 g_{m n}\right) \nabla_{p}\left(A_{q r}^{n p} \gamma^{q r} \varepsilon\right) \\
& -32 k^{3} \frac{1}{6}\left(\gamma_{m}^{p}-5 \delta_{m}^{p}\right) \nabla^{n}\left(B_{n p q r} \gamma^{q r} \varepsilon\right), \\
\tilde{D}^{m} \psi_{m}= & \gamma^{m n} \nabla_{m} \psi_{n}+k R_{m n p q} \gamma^{p q} \nabla^{n} \psi^{m} \\
& -4 k^{2} \tilde{A}^{m n}{ }_{p q} \gamma^{p q} \nabla_{n} \psi_{m} \\
& +32 k^{3} \tilde{B}_{m n p q} \gamma^{p q} \nabla^{n} \psi^{m},
\end{aligned}
$$


with

$$
\begin{aligned}
B_{p q}^{m n} \gamma^{p q} \varepsilon & =\frac{1}{6}\left(\gamma^{p[m}+5 g^{p[m}\right) \nabla^{n]} \nabla^{q}\left(A_{p q r s} \gamma^{r s} \varepsilon\right), \\
\tilde{B}_{p q}^{m n} \gamma^{p q} \varepsilon & =\tilde{A}^{p\left[{ }_{r s}\right.} \nabla_{p} \nabla^{n]} \gamma^{r s} \varepsilon .
\end{aligned}
$$

This ensures the consistency of the " $k$ family" of corrections up to seven internal derivatives.

At this order we can also return to the $\nabla R^{3}$ terms and clearly we could effectively just transpose the eleven-dimensional solutions to seven dimensions, performing minimal adjustments, as they only contain zero-forms (and 8-forms, which now vanish identically) by construction. As we saw in section 4.2.1 and appendix B, there are several such solutions, corresponding to several unfixed coefficients. A certain combination of these sevendimensional solutions will correspond to taking the eleven-dimensional $R^{4}$ action as purely internal, so their coefficients will be of zeroth order in external derivatives. Others might correspond to higher-derivative corrections beyond $R^{4}$ in eleven dimensions which factorise in such a way as to give rise to internal $R^{4}$ terms.

\subsection{Solutions with $G_{2}$ holonomy}

We will now make some considerations about the particular case when the sevendimensional manifold has $G_{2}$ holonomy.

The assumption of $G_{2}$ holonomy imposes a large number of simplifications in our formulae. We will not go through the complete solution, but note, for example, that if we take the $G_{2}$ structure to be defined by a spinor $\varepsilon$, then when evaluating our supersymmetry operators all derivatives of $\varepsilon$ drop out since $\nabla \varepsilon=0$ by $G_{2}$ holonomy.

Furthermore, we have that $R_{m n p q} \gamma^{p q} \varepsilon=0$ as well, so the entire " $k$ family" of corrections from the previous subsection vanishes identically. This can be seen either by acting on the supersymmetry variation by another $\nabla$ or introducing the $G_{2}$ invariant 3 -form and dual 4 -form in terms of the complete basis of seven-dimensional spinors given by $\left\{\varepsilon, \gamma^{m} \varepsilon\right\}$ [40]:

$$
\begin{aligned}
\gamma_{m n} \varepsilon & =\mathrm{i} \phi_{m n p} \gamma^{p} \varepsilon, \\
\gamma_{m n p} \varepsilon & =\mathrm{i} \phi_{m n p} \varepsilon-* \phi_{m n p q} \gamma^{q} \varepsilon .
\end{aligned}
$$

One finds that for $G_{2}$ holonomy manifolds the Riemann tensor satisfies $R_{m n p q} \phi^{p q r}=0$, which implies that under the $\mathbf{2 8} \rightarrow \mathbf{2 1}+\mathbf{7}$ decomposition of a 2 -form, the representation 7 is missing. This can be written equivalently as $R_{m n p q}(* \phi)^{m n}{ }_{r s}=2 R_{r s p q}$.

This brings us to the eight-derivative terms. In fact, $G_{2}$ holonomy also implies that the $t_{8} t_{8} R^{4}$ term vanishes identically [24], so the internal action actually has no corrections at order $R^{4}$. The equations of motion are still corrected [36, 41], however, and for an external flat space they become simply $\nabla^{m} \nabla_{m} Z_{n}{ }^{n}=0$, where $Z$ is a function of $R^{3}$ given below. This is then what a $G_{2}$ Lichnerowicz formula should reproduce.

There exists a well-known correction term to the supersymmetry variation for $G_{2}$ manifolds. It is usually given in terms of the $G_{2}$-invariant 3 -form $\phi_{m n p}$ as

$$
\delta \psi_{m}=\nabla_{m} \varepsilon+\mathrm{i} \alpha\left(\nabla_{n} Z_{m p}\right) \phi^{n p q} \gamma_{q} \varepsilon
$$

with $\alpha$ some real constant and

$$
Z_{m n}=g \epsilon_{m m_{1} \ldots m_{6}} \epsilon_{n n_{1} \ldots n_{6}} R^{m_{1} m_{2} n_{1} n_{2}} R^{m_{3} m_{4} n_{3} n_{4}} R^{m_{5} m_{6} n_{5} n_{6}},
$$




\begin{tabular}{|ccc|}
\hline Projection of $R^{3}$ & Rep of $G_{2}$ & Multiplicity \\
\hline$\dot{I}^{i}$ & {$[3,0]$} & 2 \\
$\dot{K}$ & {$[1,1]$} & 1 \\
$\dot{W}^{i}$ & {$[2,0]$} & 3 \\
$\dot{S}^{i}$ & {$[0,0]$} & 2 \\
\hline
\end{tabular}

Table 4. Valid embeddings of $\otimes^{3} R$ in $\delta \psi$. In each case, the index $i$ runs over the corresponding multiplicity.

is a correction term [36] which satisfies $\nabla^{m} Z_{m n}=0$ thanks to the Bianchi identity of $R_{m n p q} . Z_{m n}$ may be written in terms of our $\mathrm{SO}(7)$ bases as

$$
Z_{m n}=24\left(-\check{W}_{m n}^{1}+2 \check{W}_{m n}^{2}+\check{W}_{m n}^{3}\right)+\frac{4}{7} g_{m n}\left(\check{S}^{1}-2 \check{S}^{2}\right) .
$$

The precise form of this correction was a crucial part of the analysis of [42], which examined whether the $G_{2}$ solution remains a valid supersymmetric background to all orders of higherderivatives corrections. We will leave for future work a direct comparison of (5.11) with the reduced $x_{5}$ solution given in the previous section, but we will remark that just a quick look at the representation theory shows that it is plausible that the two match. First note that under a $G_{2}$ decomposition, the number of possible terms that can be admitted in $\delta \psi$ is quite small, they are listed in table $4 .^{9}$

We observe that since $[0,2]$ (the representation of the Weyl tensor) is not one of the admissible terms, it is not possible to obtain $R^{4}$ scalars from Lichnerowicz, which lines up with our expectation that those terms in the action vanish. Now, focusing on the terms that appear in the restricted operator (5.1), from the $s o(7)$ under $g_{2}$ branching rules we have the decompositions of the $\check{X}^{i},[0,2,0] \rightarrow[0,2]+[1,1]+[2,0]$, the $\check{V}^{i},[1,1,0] \rightarrow[1,1]+[2,0]+[0,1]$ and the $T^{i},[0,0,2] \rightarrow[2,0]+[1,0]+[0,0]$, though note that the $[1,0]$ and $[0,1]$ components drop out as they do not exist in the tensor product of $R^{3}$. The $\check{W}^{i} \in[2,0,0] \rightarrow[2,0]$ do not decompose, nor do the scalars $\check{S}^{i}$, so in particular $Z_{m n}$ keeps its form. We see that all the terms in the $x_{5}$ solution mix together in the $[1,1]+[2,0]+[0,0]$ representations, and therefore agreement with (5.11) will require the $[1,1]$ to vanish and the $[2,0]+[0,0]$ to be precisely $Z_{m n}$. A potential issue is that in eleven dimensions we ignored terms such as the ones of the type $W^{i}$ and $S^{i}$ since they did not contribute to Lichnerowicz (they do not affect the bosonic action), yet they might be required here in order to obtain a precise match at the level of the operators.

Finally, we remark that the calculation of [36] which first derived (5.11) relies on this operator satisfying an integrability condition precisely of the type that first led us to consider more general Lichnerowicz formulae, so in that sense the problem has already been solved.

\footnotetext{
${ }^{9}$ Note that here we do not require that only the antisymmetric $[\nabla, \nabla]$ appears in the $G_{2}$ Lichnerowicz formula since by using the covariantly constant spinor $(\nabla \varepsilon=0)$ we do not need to worry about tensoriality. In fact, as mentioned, the expectation is that the $G_{2}$ Lichnerowicz will result in the equation of motion $\nabla^{m} \nabla_{m} Z_{n}^{n}=0$.
} 


\subsection{Comments on compactifications}

We shall now comment on the relation between the results of subsection 5.1 and the general results of section 4 . The seven-derivative contributions to the covariant derivative $D_{a}$ are clearly related, but while for the eleven-dimensional operator these are the first higher-derivative terms, its seven-dimensional counterpart has also three- and fivederivative terms. Where do these come from?

Let us start with lowest order, i.e. the three-derivative terms in (5.3). If one thinks of $k$ not as a numerical coefficient, but a combination quadratic in external (four-dimensional) Riemann tensors, then one can find factorised terms in (4.9) and (4.10) that can yield (5.3) upon breaking eleven-dimensional Lorentz invariance.

The $y_{2}$ and $x_{5}$ families behave rather differently. $\frac{1}{2} x_{5}\left(R_{46}-4 A_{7}\right)$ appearing in (4.14) does not have any factorised terms and hence its reduction would lead directly to $k=0$. The reducible part of the $y_{2}$ family that yields a non-trivial $k$ contribution is $-\frac{1}{2} y_{2} R_{44}$.

Let us also observe that if one precipitates and uses (5.10) together with $G_{2}$ self-duality relations for the Riemann curvature, one find that the two terms proportional to $k$ cancel out in (5.4). In our approach, we keep the zero-form and the four-form separately and add the latter to the action after completing it to a top-form by wedging with $C$.

In the reduction on a $G_{2}$ holonomy manifold $X$, the relevant part of the elevendimensional action to be integrated over $X$ is given by

$$
\frac{1}{2}\left(R_{a b c d} R^{a b c d}\right)^{2}-\frac{1}{4} C \wedge \operatorname{tr} R^{2} \wedge \operatorname{tr} R^{2} \mapsto+\frac{1}{2} E_{4} \int_{X} \phi \wedge \operatorname{tr} R^{2}-\frac{1}{4} \operatorname{tr} R^{2} u_{i} \int_{X} \omega_{3}^{i} \operatorname{tr} R^{2} .
$$

Here $E_{4}$ is the four-dimensional Euler density which (up to Ricci terms) is the same as the Riemann tensor squared. In the first term on the right-hand side we have used that $R_{m n p q} R^{m n p q}=\frac{1}{2} R_{m n p q} R_{r s}{ }^{p q}(* \phi)^{m n r s}=*\left(\phi \wedge \operatorname{tr} R^{2}\right)$. Finally $\omega^{i} \in H^{3}(X)$ and $C_{3}=u_{i} \omega_{3}^{i}$, with $i=1, \cdots, b_{3}(X)$. We may decompose similarly $\phi=t_{i} \omega_{3}^{i}$. In fact $u_{i}$ and $t_{i}$ form the scalar sector of $b_{3}$ chiral superfields of the $N=1$ theory in four dimensions. We end up with a four-derivative contribution to the $N=1$ effective theory:

$$
\mathcal{L}_{N=1} \sim \alpha_{i}\left(\frac{1}{2} u_{i} E_{4}+t_{i} \operatorname{tr} R^{2}\right)
$$

with

$$
\alpha^{i}=\int_{X} \omega_{3}^{i} \wedge \operatorname{tr} R^{2}
$$

For $X=\tilde{X} \times S^{1}$, where $\tilde{X}$ is a Calabi-Yau threefold, one can recognise the familiar one-loop $R^{2}$ couplings in $N=2$ theories, where now in the internal six-dimensional integral the $\omega^{i}$ are replaced by the forms in the $H^{(1,1)}(\tilde{X})$, and $u_{i}+\mathrm{i} t_{i}$ is the complex scalar in the $N=2$ vector multiplets [43].

As mentioned earlier and further elaborated below, the terms in the action with $k$ factors should not receive corrections from higher-derivative (higher string loop terms) and hence we do not expect the coupling (5.15) to receive further perturbative corrections. We may remark that the moduli spaces of $G_{2}$ compactifications are not factorised and have 
(complex) dimension $b_{3}(X)+b_{2}(X) \cdot{ }^{10}$ It is curious that the higher-derivative couplings make use of $b_{3}(X)$ topological numbers $\alpha^{i}$ and distinguish between the two sectors.

We can turn to the next order - five derivative terms in the operators. Note that these are designed to cancel non-tensorial terms in (5.5). However, they contain a factor of $k^{2}$ on top of two Riemann tensors and two $\nabla$, and so from the eleven-dimensional point of view are order $R^{7}$. Hence the additional contributions to the supersymmetry operators are reductions of the next order terms in eleven dimensions, i.e. 13 derivative terms. Similarly, at the next order of internal derivatives we have terms $\sim k^{3}$, and these come from the reduction of 19 derivative corrections to eleven-dimensional supersymmetry. Note that we are only probing the fraction of the higher (than seven) derivative terms in supersymmetry whose purpose is to cancel unwanted non-tensorial contributions from cross-terms of lowerderivative contributions. By design the action itself stays order $\sim R^{4}$. Notice, however, that the seven-dimensional Lichnerowicz method seems to be giving information about terms that are higher order in the eleven-dimensional sense.

We may finally comment on factorisability properties of higher derivative terms. In [27] the terms that allowed to factor out a single Ricci scalar were discussed. Here we completely ignore the Ricci terms, so we cannot further comment on corrections of that type. However, our seven-dimensional calculation would appear to rule out certain other types of terms in eleven dimensions. Consider, for example, an order $R^{7}$ correction to the action that factorises as $R^{3} \cdot R_{44}$, where $R^{3}$ is some linear combination of the $S^{i}$ given in appendix A.1 and $R_{44}$ was given in (4.13). Upon decomposing to seven dimensions, this would lead to a term $\tilde{k}\left(R^{7 \mathrm{~d}}\right)^{3}$ in the reduced action, with $\tilde{k}=R_{44}^{4 \mathrm{~d}}=\left(R_{\mu \nu \lambda \rho} R^{\mu \nu \lambda \rho}\right)^{2}$. This would be in contradiction with our Lichnerowicz calculation in section 5.1.3 which disallowed $R^{3}$ terms in seven dimensions. Thus, we are lead to conclude that corrections of the type $R^{3}$. [anything nonzero in $4 \mathrm{~d}$ ] appear to be ruled out in eleven dimensions. On the other hand, a pure Riemann term like $R^{3} \cdot\left(\epsilon_{8} \epsilon_{8} R^{4}\right)$ is possible. Similar considerations also imply that (beyond order $R^{4}$ ) terms that factorise as $R^{2} \cdot$ [anything nonzero in $4 \mathrm{~d}$ ] are likewise ruled out.

\section{$6 \quad$ Future directions}

The effects induced by higher-derivative corrections play an important role in many lower dimensional theories arising from compactifications. It may be useful, even if more laborious, to work out a full reduction of the eleven-dimensional Lichnerowicz formula, rather than study a lower dimensional "descendant", as we did here. An intriguing aspect of studying the formula on a product of internal and external spaces is that different derivative orders get mixed on a lower dimensional component, allowing for glimpses into further corrections to supersymmetry operators. There are still open problems concerning the eleven-derivative covariant couplings, and we conclude by mentioning two venues of possible progress.

\footnotetext{
${ }^{10}$ We discussed here the $b_{3}(X)$ chiral multiplets made of deformations of the metric and the scalar modes coming from $C_{3}$. The latter also yields $b_{2}(X)$ vector fields, which make the bosonic part of vector multiplets.
} 


\subsection{Adding fluxes}

We began by showing that the classical supersymmetry operators with flux obey a Lichnerowicz relation that reproduces the classical action. However, when we moved to the higher-order corrections we set $G=0$ in order to simplify the computation. A clear next step is to restore those terms and obtain the flux completion of $R^{4}$.

The issue of computing the full set of $G$ flux contributions to the eight derivative corrections is a long standing one. Progress has been made in string theory, and it can be shown that at one loop most of the NS sector contributions are captured by introducing a connection with torsion $\omega^{\mathrm{LC}} \rightarrow \omega^{\mathrm{LC}}+H$. There are, however, additional ambiguities associated with lifting to eleven dimensions, and replacing $H$ by $G$ [44].

In the context of the M-theoretic Lichnerowicz method, the computation of the flux terms should proceed in a straightforward iterative manner. Firstly, promoting the leading term $\nabla_{a} \rightarrow \nabla_{a}^{G}$ in the supersymmetry operator $D_{a}$ will immediately break the tensoriality of $\tilde{D}^{a} D_{a} \varepsilon$ and will require adjustments to the existing higher-derivative terms - for example, all the $\nabla_{a}$ in the correction terms of $\tilde{D}_{a}$ will likewise have to be replaced by $\nabla_{a}^{G}$. Additionally, we neglected Ricci terms but now they become proportional to $G^{2}$ by the equations of motion so they cannot be ignored. Instead, we have that factors of $\gamma^{a}\left[\nabla_{a}^{G}, \nabla_{b}^{G}\right]$ and $\left(\tilde{\nabla}^{G}\right)^{a} \nabla_{a}^{G}$ give the combinations of covariant derivatives that vanish on-shell. Finally, it will be necessary to impose the constraint that the M-theory Lichnerowicz define just a scalar and an 8-form. This will require the addition of new terms to the corrected operators to cancel the other $p$-forms, which should be easier to do if one proceeds order by order in powers of the $G$ flux.

Note that at four-derivative order, the couplings in the seven-dimensional theory with 16 supercharges including four-form flux are known without ambiguities [44]. This case should provide a good test for completing the seven-dimensional Lichnerowicz formula with $G$.

\subsection{Towards $R^{7}$}

The M-theoretic Lichnerowicz computed from the operators we defined in section 4.2.1 $\tilde{D}^{a} D_{a} \varepsilon$ only results in a scalar and a 3 -form up to order $R^{4}$. If we include the higher order terms in the computation this will fail, and the form of this failure is clear - if we schematically write the corrections $D=\nabla+\nabla R^{3}$ and $\tilde{D}=\nabla+R^{3} \nabla$, then the full $\tilde{D} D$ will include $R^{3} \nabla \nabla R^{3}$, which will even involve non-tensor terms. These terms are of the same order as $R^{7}$, so we are led to conclude that no new corrections will be needed at order $R^{5}$ or $R^{6}$, but are necessary at $R^{7}$. This is consistent with the fact that in the strong coupling eleven-dimensional limit [45], the only surviving terms will be of order $R^{3 l+1}$ for loop order $l$.

In principle, the Lichnerowicz procedure should allow us to deduce what is the form of these corrections. However, computationally it is significantly more difficult to generate the necessary tensor products of seven Riemann tensors, and, in addition, it is expected that at this order there might be terms involving explicit connections $\nabla$ in the action, further complicating matters. Nevertheless, since we can easily compute the 'problem' terms explicitly in the Lichnerowicz equation, it seems hopeful that one can cancel them 
step-by-step by suitable modifications of the $D$ and $\tilde{D}$ operators, just like we did in the simpler seven-dimensional case in section 5.1.2. As an example, consider just the $X^{1}$ part of the $y_{2}$ solution for the operators (4.9), i.e.

$$
\begin{aligned}
D_{a} \varepsilon & =\nabla_{a} \varepsilon-y_{2} \nabla^{b}\left(X_{a b c d}^{1} \gamma^{c d} \varepsilon\right)+\ldots \\
& =\nabla_{a} \varepsilon-y_{2} \nabla^{b}\left(R^{2} R_{a b c d} \gamma^{c d} \varepsilon\right)+\ldots \\
\tilde{D}^{a} \psi_{a} & =\gamma^{a b} \nabla_{a} \psi_{b}-y_{2} X_{a b c d}^{1} \gamma^{c d} \nabla^{b} \psi^{a}+\ldots \\
& =\gamma^{a b} \nabla_{a} \psi_{b}-y_{2} R^{2} R_{a b c d} \gamma^{c d} \nabla^{b} \psi^{a}+\ldots
\end{aligned}
$$

which gives in the M-theory Lichnerowicz

$$
\tilde{D}^{a} D_{a} \varepsilon=\cdots+\left(y_{2}\right)^{2} R^{2} R_{b c d}^{a} \gamma^{c d} \nabla^{b} \nabla^{e}\left(R^{2} R_{a e f g} \gamma^{f g} \varepsilon\right)+\ldots
$$

This term is analogous to the one we found in seven dimensions, and so can be cancelled in a similar manner. We therefore expect that there will be a correction to the supersymmetry operators at 13 derivatives given by

$$
\begin{aligned}
D_{a} \varepsilon= & \nabla_{a} \varepsilon-y_{2} \nabla^{b}\left(R^{2} R_{a b c d} \gamma^{c d} \varepsilon\right) \\
& +4\left(y_{2}\right)^{2} \frac{1}{10}\left(\gamma_{a b}-9 g_{a b}\right) \nabla_{c}\left(R^{2} \nabla^{[b} \nabla_{d}\left(R^{c] d}{ }_{e f} R^{2} \gamma^{e f} \varepsilon\right)\right)+\ldots \\
\tilde{D}^{a} \psi_{a}= & \gamma^{a b} \nabla_{a} \psi_{b}-y_{2} R^{2} R_{a b c d} \gamma^{c d} \nabla^{b} \psi^{a} \\
& -4\left(y_{2}\right)^{2} R^{c[a}{ }_{e f} R^{2} \nabla_{c} \nabla^{b]} \gamma^{e f}\left(R^{2} \nabla_{b} \psi_{a}\right)+\ldots
\end{aligned}
$$

Since the effect of this particular correction is to precisely cancel the non-tensorial piece in (6.2), it does not generate new contributions to the action of order $R^{7}$.

\section{Acknowledgments}

We would like to thank K. Becker, D. Robbins, A. Royston, P. Vanhove for useful discussions. A. C. has been supported by the Laboratoire d'Excellence CARMIN. A. C. also thanks the Program on the Mathematics of String Theory at Institut Henri Poincaré, Paris for hospitality during the completion of this work. The work of R. M. is supported in part by the Agence Nationale de la Recherche under the grant 12-BS05-003-01.

\section{A Projectors for $R^{3}$}

\section{A.1 In eleven dimensions}

Here we present bases for projections of tensor products of Weyl tensors into irreducible representations of $\mathrm{SO}(10,1)$. Throughout, same letter indices which are free are assumed to be antisymmetrised with unit weight (contracted indices have no such assumption). We will be considering the irreducible representations listed in table 2 which are relevant for the Lichnerowicz calculation. For convenience, these are reproduced in table 5. 


\begin{tabular}{|ccc|}
\hline Projection of $R^{3}$ & Rep of $\mathrm{SO}(10,1)$ & Multiplicity \\
\hline$X^{i}$ & {$[0,2,0,0,0]$} & 8 \\
$W^{i}$ & {$[2,0,0,0,0]$} & 3 \\
$S^{i}$ & {$[0,0,0,0,0]$} & 2 \\
\hline$Y^{i}$ & {$[0,1,0,0,2]$} & 2 \\
$V^{i}$ & {$[1,0,0,0,2]$} & 2 \\
$T^{i}$ & {$[0,0,0,1,0]$} & 3 \\
\hline$Z^{i}$ & {$[0,1,0,1,0]$} & 3 \\
$U^{i}$ & {$[1,0,1,0,0]$} & 3 \\
\hline
\end{tabular}

Table 5. Relevant projections of $\otimes^{3} R$ in eleven dimensions. In each case, the index $i$ runs over the corresponding multiplicity.

The four-index $X^{i}$ terms contain two pairs of antisymmetric indices which are symmetric under exchange, and are fully traceless:

$$
\begin{aligned}
& X_{a_{1} a_{2} b_{1} b_{2}}^{1}=R_{a_{1} a_{2} b_{1} b_{2}} R_{c_{1} c_{2} d_{1} d_{2}} R_{c_{1} c_{2} d_{1} d_{2}}, \\
& X_{a_{1} a_{2} b_{1} b_{2}}^{2}=\frac{1}{2} R_{a_{1} a_{2} b_{1} c_{1}} R_{b_{2} c_{2} d_{1} d_{2}} R_{c_{1} c_{2} d_{1} d_{2}}+\frac{1}{2} R_{b_{1} b_{2} a_{1} c_{1}} R_{a_{2} c_{2} d_{1} d_{2}} R_{c_{1} c_{2} d_{1} d_{2}} \\
& +\frac{2}{9} g_{a_{2} b_{1}} R_{b_{2} d_{1} a_{1} d_{2}} R_{d_{1} c_{1} c_{2} e_{1}} R_{d_{2} c_{1} c_{2} e_{1}}, \\
& X_{a_{1} a_{2} b_{1} b_{2}}^{3}=\frac{2}{3} R_{a_{1} a_{2} c_{1} c_{2}} R_{b_{1} b_{2} d_{1} d_{2}} R_{c_{1} c_{2} d_{1} d_{2}}-\frac{2}{3} R_{a_{1} b_{1} c_{1} c_{2}} R_{b_{2} a_{2} d_{1} d_{2}} R_{c_{1} c_{2} d_{1} d_{2}} \\
& +\frac{4}{9} g_{a_{2} b_{1}} R_{b_{2} e_{1} d_{1} d_{2}} R_{d_{1} d_{2} c_{1} c_{2}} R_{c_{1} c_{2} a_{1} e_{1}} \\
& -\frac{1}{45} g_{a_{2} b_{1}} g_{a_{1} b_{2}} R_{d_{1} d_{2} e_{1} e_{2}} R_{e_{1} e_{2} c_{1} c_{2}} R_{c_{1} c_{2} d_{1} d_{2}}, \\
& X_{a_{1} a_{2} b_{1} b_{2}}^{4}=\frac{2}{3} R_{a_{1} c_{1} b_{1} c_{2}} R_{a_{2} d_{1} b_{2} d_{2}} R_{c_{1} d_{1} c_{2} d_{2}}+\frac{1}{3} R_{a_{1} c_{1} b_{1} c_{2}} R_{a_{2} d_{1} b_{2} d_{2}} R_{c_{1} d_{2} c_{2} d_{1}} \\
& +\frac{1}{24} R_{a_{1} a_{2} c_{1} c_{2}} R_{b_{1} b_{2} d_{1} d_{2}} R_{c_{1} c_{2} d_{1} d_{2}} \\
& +\frac{1}{18} g_{a_{2} b_{1}} R_{b_{2} e_{1} d_{1} d_{2}} R_{d_{1} d_{2} c_{1} c_{2}} R_{c_{1} c_{2} a_{1} e_{1}}-\frac{2}{9} g_{a_{2} b_{1}} R_{b_{2} d_{1} e_{1} d_{2}} R_{d_{1} c_{1} d_{2} c_{2}} R_{c_{1} a_{1} c_{2} e_{1}} \\
& -\frac{1}{360} g_{a_{2} b_{1}} g_{a_{1} b_{2}} R_{d_{1} d_{2} b 3 b 4} R_{b 3 b 4 c_{1} c_{2}} R_{c_{1} c_{2} d_{1} d_{2}}+\frac{1}{90} g_{a_{2} b_{1}} g_{a_{1} b_{2}} R_{d_{1} e_{1} d_{2} e_{2}} R_{e_{1} c_{1} e_{2} c_{2}} R_{c_{1} d_{1} c_{2} d_{2}} \text {, } \\
& X_{a_{1} a_{2} b_{1} b_{2}}^{5}=\frac{1}{3} R_{a_{1} a_{2} c_{1} c_{2}} R_{b_{1} c_{1} d_{1} d_{2}} R_{b_{2} c_{2} d_{1} d_{2}}+\frac{1}{3} R_{b_{1} b_{2} c_{1} c_{2}} R_{a_{1} c_{1} d_{1} d_{2}} R_{a_{2} c_{2} d_{1} d_{2}} \\
& -\frac{2}{3} R_{a_{1} b_{1} c_{1} c_{2}} R_{b_{2} c_{1} d_{1} d_{2}} R_{a_{2} c_{2} d_{1} d_{2}} \\
& +\frac{2}{9} g_{a_{2} b_{1}} R_{b_{2} e_{1} d_{1} d_{2}} R_{d_{1} d_{2} c_{1} c_{2}} R_{c_{1} c_{2} a_{1} e_{1}} \\
& -\frac{1}{90} g_{a_{2} b_{1}} g_{a_{1} b_{2}} R_{d_{1} d_{2} e_{1} e_{2}} R_{e_{1} e_{2} c_{1} c_{2}} R_{c_{1} c_{2} d_{1} d_{2}},
\end{aligned}
$$




$$
\begin{aligned}
& X_{a_{1} a_{2} b_{1} b_{2}}^{6}=\frac{2}{3} R_{a_{1} c_{1} b_{1} c_{2}} R_{a_{2} d_{1} c_{1} d_{2}} R_{b_{2} d_{1} c_{2} d_{2}}+\frac{1}{3} R_{a_{1} c_{2} b_{1} c_{1}} R_{a_{2} d_{1} c_{1} d_{2}} R_{b_{2} d_{1} c_{2} d_{2}} \\
& +\frac{1}{12} R_{a_{1} a_{2} c_{1} c_{2}} R_{b_{1} d_{1} c_{1} d_{2}} R_{b_{2} d_{1} c_{2} d_{2}}+\frac{1}{12} R_{b_{1} b_{2} c_{1} c_{2}} R_{a_{1} d_{1} c_{1} d_{2}} R_{a_{2} d_{1} c_{2} d_{2}} \\
& -\frac{1}{18} g_{a_{2} b_{1}} R_{b_{2} e_{1} d_{1} d_{2}} R_{d_{1} d_{2} c_{1} c_{2}} R_{c_{1} c_{2} a_{1} e_{1}}+\frac{2}{9} g_{a_{2} b_{1}} R_{b_{2} d_{1} e_{1} d_{2}} R_{d_{1} c_{1} d_{2} c_{2}} R_{c_{1} a_{1} c_{2} e_{1}} \\
& +\frac{1}{9} g_{a_{2} b_{1}} R_{b_{2} d_{1} a_{1} d_{2}} R_{d_{1} c_{1} c_{2} e_{1}} R_{d_{2} c_{1} c_{2} e_{1}} \\
& +\frac{1}{360} g_{a_{2} b_{1}} g_{a_{1} b_{2}} R_{d_{1} d_{2} e_{1} e_{2}} R_{e_{1} e_{2} c_{1} c_{2}} R_{c_{1} c_{2} d_{1} d_{2}}-\frac{1}{90} g_{a_{2} b_{1}} g_{a_{1} b_{2}} R_{d_{1} e_{1} d_{2} e_{2}} R_{e_{1} c_{1} e_{2} c_{2}} R_{c_{1} d_{1} c_{2} d_{2}}, \\
& X_{a_{1} a_{2} b_{1} b_{2}}^{7}=\frac{2}{3} R_{a_{1} c_{1} b_{1} c_{2}} R_{a_{2} c_{2} d_{1} d_{2}} R_{b_{2} c_{1} d_{1} d_{2}}+\frac{1}{3} R_{a_{1} c_{2} b_{1} c_{1}} R_{a_{2} c_{2} d_{1} d_{2}} R_{b_{2} c_{1} d_{1} d_{2}} \\
& -\frac{1}{12} R_{a_{1} a_{2} c_{1} c_{2}} R_{b_{1} c_{1} d_{1} d_{2}} R_{b_{2} c_{2} d_{1} d_{2}}-\frac{1}{12} R_{b_{1} b_{2} c_{1} c_{2}} R_{a_{1} c_{1} d_{1} d_{2}} R_{a_{2} c_{2} d_{1} d_{2}} \\
& -\frac{2}{9} g_{a_{2} b_{1}} R_{b_{2} e_{1} d_{1} d_{2}} R_{d_{1} d_{2} c_{1} c_{2}} R_{c_{1} c_{2} a_{1} e_{1}}+\frac{1}{9} g_{a_{2} b_{1}} R_{b_{2} d_{1} a_{1} d_{2}} R_{d_{1} c_{1} c_{2} e_{1}} R_{d_{2} c_{1} c_{2} e_{1}} \\
& +\frac{1}{90} g_{a_{2} b_{1}} g_{a_{1} b_{2}} R_{d_{1} d_{2} e_{1} e_{2}} R_{e_{1} e_{2} 4 c_{1} c_{2}} R_{c_{1} c_{2} d_{1} d_{2}}, \\
& X_{a_{1} a_{2} b_{1} b_{2}}^{8}=\frac{1}{3} R_{a_{1} a_{2} c_{1} c_{2}} R_{b_{1} d_{1} c_{1} d_{2}} R_{b_{2} d_{1} c_{2} d_{2}}+\frac{1}{3} R_{b_{1} b_{2} c_{1} c_{2}} R_{a_{1} d_{1} c_{1} d_{2}} R_{a_{2} d_{1} c_{2} d_{2}} \\
& -\frac{2}{3} R_{a_{1} b_{1} c_{1} c_{2}} R_{b_{2} d_{1} c_{1} d_{2}} R_{a_{2} d_{1} c_{2} d_{2}} \\
& +\frac{4}{9} g_{a_{2} b_{1}} R_{b_{2} d_{1} e_{1} d_{2}} R_{d_{1} c_{1} d_{2} c_{2}} R_{c_{1} a_{1} c_{2} e_{1}} \\
& -\frac{1}{45} g_{a_{2} b_{1}} g_{a_{1} b_{2}} R_{d_{1} e_{1} d_{2} e_{2}} R_{e_{1} c_{1} e_{2} c_{2}} R_{c_{1} d_{1} c_{2} d_{2}} \text {. }
\end{aligned}
$$

The two-index $W^{i}$ are symmetric traceless:

$$
\begin{aligned}
& W_{a_{1} b_{1}}^{1}=R_{a_{1} d_{1} e_{1} e_{2}} R_{e_{1} e_{2} c_{1} c_{2}} R_{c_{1} c_{2} b_{1} d_{1}}-\frac{1}{11} g_{a_{1} b_{1}} R_{e_{1} e_{2} d_{2} b_{2}} R_{d_{2} b_{2} c_{1} c_{2}} R_{c_{1} c_{2} e_{1} e_{2}}, \\
& W_{a_{1} b_{1}}^{2}=R_{a_{1} e_{1} d_{1} e_{2}} R_{e_{1} c_{1} e_{2} c_{2}} R_{c_{1} b_{1} c_{2} d_{1}}-\frac{1}{11} g_{a_{1} b_{1}} R_{e_{1} a_{2} e_{2} b_{2}} R_{a_{2} c_{1} b_{2} c_{2}} R_{c_{1} e_{1} c_{2} e_{2}}, \\
& W_{a_{1} b_{1}}^{3}=R_{a_{1} e_{1} b_{1} c_{1}} R_{e_{1} e_{2} d_{1} d_{2}} R_{c_{1} e_{2} d_{1} d_{2}} .
\end{aligned}
$$

The $S^{i}$ are scalars:

$$
\begin{aligned}
& S^{1}=R_{a_{1} a_{2} b_{1} b_{2}} R_{b_{1} b_{2} c_{1} c_{2}} R_{c_{1} c_{2} a_{1} a_{2}}, \\
& S^{2}=R_{a_{1} b_{1} a_{2} b_{2}} R_{b_{1} c_{1} b_{2} c_{2}} R_{c_{1} a_{1} c_{2} a_{2}} .
\end{aligned}
$$

The eight-index $Y^{i}$ have a pair of antisymmetric indices, another set of six antisymmetric indices, vanish if any seven indices are antisymmetrised, and are traceless:

$$
\begin{aligned}
& Y_{a_{1} a_{2} b_{1} \ldots b_{6}}^{1}= R_{a_{1} a_{2} b_{1} b_{2}} R_{b_{3} b_{4} d_{1} d_{2}} R_{b_{5} b_{6} d_{1} d_{2}} \\
&-\frac{8}{5} g_{a_{1} b_{1}} R_{c_{1} a_{2} b_{2} b_{3}} R_{c_{1} b_{4} d_{1} d_{2}} R_{b_{5} b_{6} d_{1} d_{2}} \\
&+\frac{2}{15} g_{a_{1} b_{1}} g_{a_{2} b_{2}} R_{c_{1} c_{2} b_{3} b_{4}} R_{d_{1} d_{2} b_{5} b_{6}} R_{c_{1} c_{2} d_{1} d_{2}}-\frac{4}{15} g_{a_{1} b_{1}} g_{a_{2} b_{2}} R_{c_{1} c_{2} b_{3} b_{4}} R_{c_{1} b_{5} d_{1} d_{2}} R_{c_{2} b_{6} d_{1} d_{2}}, \\
& Y_{a_{1} a_{2} b_{1} \ldots b_{6}}^{2}= R_{a_{1} c_{1} b_{1} b_{2}} R_{a_{2} c_{2} b_{3} b_{4}} R_{b_{5} b_{6} c_{1} c_{2}} \\
&-\frac{2}{5} g_{a_{1} b_{1}} R_{c_{1} a_{2} b_{2} b_{3}} R_{c_{1} b_{4} d_{1} d_{2}} R_{b_{5} b_{6} d_{1} d_{2}}-\frac{4}{5} g_{a_{1} b_{1}} R_{c_{1} d_{1} b_{3} b_{4}} R_{a_{2} d_{2} c_{1} b_{2}} R_{d_{1} d_{2} b_{5} b_{6}}
\end{aligned}
$$




$$
\begin{gathered}
+\frac{1}{30} g_{a_{1} b_{1}} g_{a_{2} b_{2}} R_{c_{1} c_{2} b_{3} b_{4}} R_{d_{1} d_{2} b_{5} b_{6}} R_{c_{1} c_{2} d_{1} d_{2}}-\frac{2}{15} g_{a_{1} b_{1}} g_{a_{2} b_{2}} R_{c_{1} c_{2} b_{3} b_{4}} R_{c_{1} b_{5} d_{1} d_{2}} R_{c_{2} b_{6} d_{1} d_{2}} \\
+\frac{2}{15} g_{a_{1} b_{1}} g_{a_{2} b_{2}} R_{d_{1} d_{2} b_{3} b_{4}} R_{c_{1} d_{1} c_{2} b_{5}} R_{c_{2} d_{2} c_{1} b_{6}} .
\end{gathered}
$$

The six-index $V^{i}$ have a set of five antisymmetric indices, vanish if the six indices are antisymmetrised, and are traceless:

$$
\begin{aligned}
V_{a_{2} b_{1} \ldots b_{5}}^{1}= & R_{c_{1} a_{2} b_{2} b_{3}} R_{c_{1} b_{4} d_{1} d_{2}} R_{b_{5} b_{1} d_{1} d_{2}} \\
& -\frac{1}{7} g_{a_{2} b_{2}} R_{c_{1} c_{2} b_{5} b_{1}} R_{d_{1} d_{2} b_{3} b_{4}} R_{c_{1} c_{2} d_{1} d_{2}}+\frac{2}{7} g_{a_{2} b_{2}} R_{c_{1} c_{2} b_{5} b_{1}} R_{d_{1} d_{2} c_{1} b_{3}} R_{d_{1} d_{2} c_{2} b_{4}}, \\
V_{a_{2} b_{1} \ldots b_{5}}^{2}= & R_{c_{1} d_{1} b_{3} b_{4}} R_{a_{2} d_{2} c_{1} b_{2}} R_{d_{1} d_{2} b_{5} b_{1}} \\
& -\frac{1}{7} g_{a_{2} b_{2}} R_{c_{1} c_{2} b_{5} b_{1}} R_{d_{1} d_{2} c_{1} b_{3}} R_{d_{1} d_{2} c_{2} b_{4}}-\frac{2}{7} g_{a_{2} b_{2}} R_{d_{1} d_{2} b_{5} b_{1}} R_{c_{1} d_{1} c_{2} b_{3}} R_{c_{2} d_{2} c_{1} b_{4}} .
\end{aligned}
$$

The $T^{i}$ are 4-forms:

$$
\begin{aligned}
& T_{b_{1} \ldots b_{4}}^{1}=R_{c_{1} c_{2} b_{1} b_{2}} R_{d_{1} d_{2} b_{3} b_{4}} R_{c_{1} c_{2} d_{1} d_{2}}, \\
& T_{b_{1} \ldots b_{4}}^{2}=R_{c_{1} c_{2} b_{1} b_{2}} R_{d_{1} d_{2} c_{1} b_{3}} R_{d_{1} d_{2} c_{2} b_{4}}, \\
& T_{b_{1} \ldots b_{4}}^{3}=R_{d_{1} d_{2} b_{1} b_{2}} R_{c_{1} d_{1} c_{2} b_{3}} R_{c_{2} d_{2} c_{1} b_{4}} .
\end{aligned}
$$

The six-index $Z^{i}$ have a pair of antisymmetric indices, another set of four antisymmetric indices, vanish if any five indices are antisymmetrised, and are traceless:

$$
\begin{aligned}
& Z_{a_{1} a_{2} b_{1} \ldots b_{4}}^{1}=\frac{1}{2} R_{a_{1} a_{2} d_{1} b_{1}} R_{c_{1} c_{2} d_{1} b_{2}} R_{c_{1} c_{2} b_{3} b_{4}}+\frac{1}{2} R_{b_{2} b_{1} d_{1} a_{2}} R_{c_{1} c_{2} d_{1} a_{1}} R_{c_{1} c_{2} b_{3} b_{4}} \\
& +\frac{1}{7} g_{a_{2} b_{4}} R_{a_{1} c_{1} b_{1} b_{2}} R_{d_{1} d_{2} c_{2} c_{1}} R_{d_{1} d_{2} c_{2} b_{3}}+\frac{1}{7} g_{a_{2} b_{4}} R_{c_{1} c_{2} b_{1} b_{2}} R_{c_{1} b_{3} d_{1} d_{2}} R_{d_{1} d_{2} c_{2} a_{1}} \\
& +\frac{1}{7} g_{a_{2} b_{4}} R_{c_{1} c_{2} a_{1} b_{2}} R_{c_{1} b_{3} d_{1} d_{2}} R_{d_{1} d_{2} c_{2} b_{1}} \\
& Z_{a_{1} a_{2} b_{1} \ldots b_{4}}^{2}=\frac{1}{2} R_{a_{1} c_{1} d_{1} b_{1}} R_{c_{1} c_{2} d_{1} b_{2}} R_{a_{2} c_{2} b_{3} b_{4}}+\frac{1}{2} R_{a_{1} d_{1} c_{1} b_{1}} R_{c_{1} c_{2} d_{1} b_{2}} R_{a_{2} c_{2} b_{3} b_{4}} \\
& -\frac{3}{28} g_{a_{2} b_{4}} R_{a_{1} c_{1} b_{1} b_{2}} R_{d_{1} d_{2} c_{2} c_{1}} R_{d_{1} d_{2} c_{2} b_{3}}-\frac{1}{28} g_{a_{2} b_{4}} R_{c_{1} c_{2} b_{1} b_{2}} R_{c_{1} b_{3} d_{1} d_{2}} R_{d_{1} d_{2} c_{2} a_{1}} \\
& -\frac{1}{28} g_{a_{2} b_{4}} R_{c_{1} c_{2} a_{1} b_{2}} R_{c_{1} b_{3} d_{1} d_{2}} R_{d_{1} d_{2} c_{2} b_{1}}+\frac{1}{7} g_{a_{2} b_{4}} R_{c_{1} c_{2} b_{1} b_{2}} R_{c_{1} d_{1} b_{3} d_{2}} R_{d_{1} c_{2} d_{2} a_{1}} \\
& +\frac{1}{7} g_{a_{2} b_{4}} R_{c_{1} c_{2} a_{1} b_{2}} R_{c_{1} d_{1} b_{3} d_{2}} R_{d_{1} c_{2} d_{2} b_{1}} \text {, } \\
& Z_{a_{1} a_{2} b_{1} \ldots b_{4}}^{3}=\frac{1}{2} R_{a_{1} d_{1} c_{1} c_{2}} R_{c_{1} c_{2} b_{1} b_{2}} R_{a_{2} d_{1} b_{3} b_{4}}+\frac{1}{2} R_{b_{1} d_{1} c_{1} c_{2}} R_{c_{1} c_{2} a_{1} b_{2}} R_{a_{2} d_{1} b_{3} b_{4}} \\
& -\frac{3}{14} g_{a_{2} b_{4}} R_{a_{1} c_{1} b_{1} b_{2}} R_{d_{1} d_{2} c_{2} c_{1}} R_{d_{1} d_{2} c_{2} b_{3}}-\frac{1}{14} g_{a_{2} b_{4}} R_{c_{1} c_{2} b_{1} b_{2}} R_{c_{1} b_{3} d_{1} d_{2}} R_{d_{1} d_{2} c_{2} a_{1}} \\
& -\frac{1}{14} g_{a_{2} b_{4}} R_{c_{1} c_{2} a_{1} b_{2}} R_{c_{1} b_{3} d_{1} d_{2}} R_{d_{1} d_{2} c_{2} b_{1}} \text {. }
\end{aligned}
$$




\begin{tabular}{|ccc|}
\hline Projection of $R^{3}$ & Rep of SO(7) & Multiplicity \\
\hline$\check{X}^{i}$ & {$[0,2,0]$} & 7 \\
$\check{W}^{i}$ & {$[2,0,0]$} & 3 \\
$\check{S}^{i}$ & {$[0,0,0]$} & 2 \\
\hline$\check{V}^{i}$ & {$[1,1,0]$} & 2 \\
$\check{T}^{i}$ & {$[0,0,2]$} & 3 \\
\hline$\check{Z}^{i}$ & {$[0,1,2]$} & 3 \\
$\check{U}^{i}$ & {$[1,0,2]$} & 3 \\
\hline$\check{L}^{i}$ & {$[2,1,0]$} & 3 \\
$\check{M}^{i}$ & {$[2,0,2]$} & 6 \\
\hline
\end{tabular}

Table 6. Valid embeddings of $\otimes^{3} R$ in $\delta \psi$. In each case, the index $i$ runs over the corresponding multiplicity.

The four-index $U^{i}$ have a set of three antisymmetric indices, vanish if the four indices are antisymmetrised, and are traceless:

$$
\begin{aligned}
U_{a_{1} b_{1} b_{2} b_{3}}^{1} & =R_{a_{1} c_{1} b_{1} b_{2}} R_{d_{1} d_{2} c_{2} c_{1}} R_{d_{1} d_{2} c_{2} b_{3}}, \\
U_{a_{1} b_{1} b_{2} b_{3}}^{2} & =\frac{1}{2} R_{c_{1} c_{2} b_{1} b_{2}} R_{c_{1} d_{1} b_{3} d_{2}} R_{d_{1} c_{2} d_{2} a_{1}}+\frac{1}{2} R_{c_{1} c_{2} a_{1} b_{2}} R_{c_{1} d_{1} b_{3} d_{2}} R_{d_{1} c_{2} d_{2} b_{1}}, \\
U_{a_{1} b_{1} b_{2} b_{3}}^{3} & =\frac{1}{2} R_{c_{1} c_{2} b_{1} b_{2}} R_{c_{1} b_{3} d_{1} d_{2}} R_{d_{1} d_{2} c_{2} a_{1}}+\frac{1}{2} R_{c_{1} c_{2} a_{1} b_{2}} R_{c_{1} b_{3} d_{1} d_{2}} R_{d_{1} d_{2} c_{2} b_{1}} .
\end{aligned}
$$

\section{A.2 In seven dimensions}

In principle, the seven-dimensional supersymmetry operator can contain any of the $\mathrm{SO}(7)$ representations listed in table 6 . However, we write explicitly only the projections that will be relevant for a reduction of the $x_{5}$ solution in eleven dimensions from section 4.2.1.

Indices $m, n \ldots$ run from 1 to 7 , and, as before, any free indices with the same letter are assumed to be antisymmetrised. The metric $g$ is now that of the internal seven-dimensional space.

We will consider two $\check{X}^{i}$ terms. They contain two pairs of antisymmetric indices which are symmetric under exchange, and are fully traceless:

$$
\begin{aligned}
\check{X}_{m_{1} m_{2} n_{1} n_{2}}^{5}= & \frac{1}{3} R_{m_{1} m_{2} p_{1} p_{2}} R_{n_{1} p_{1} q_{1} q_{2}} R_{n_{2} p_{2} q_{1} q_{2}}+\frac{1}{3} R_{n_{1} n_{2} p_{1} p_{2}} R_{m_{1} p_{1} q_{1} q_{2}} R_{m_{2} p_{2} q_{1} q_{2}} \\
& -\frac{2}{3} R_{m_{1} n_{1} p_{1} p_{2}} R_{n_{2} p_{1} q_{1} q_{2}} R_{m_{2} p_{2} q_{1} q_{2}} \\
& +\frac{2}{5} g_{m_{2} n_{1}} R_{n_{2} r_{1} q_{1} q_{2}} R_{q_{1} q_{2} p_{1} p_{2}} R_{p_{1} p_{2} m_{1} r_{1}} \\
& -\frac{1}{30} g_{m_{2} n_{1}} g_{m_{1} n_{2}} R_{q_{1} q_{2} r_{1} r_{2}} R_{r_{1} r_{2} p_{1} p_{2}} R_{p_{1} p_{2} q_{1} q_{2}}, \\
\check{X}_{m_{1} m_{2} n_{1} n_{2}}^{6}= & \frac{2}{3} R_{m_{1} p_{1} n_{1} p_{2}} R_{m_{2} q_{1} p_{1} q_{2}} R_{n_{2} q_{1} p_{2} q_{2}}+\frac{1}{3} R_{m_{1} p_{2} n_{1} p_{1}} R_{m_{2} q_{1} p_{1} q_{2}} R_{n_{2} q_{1} p_{2} q_{2}} \\
& +\frac{1}{12} R_{m_{1} m_{2} p_{1} p_{2}} R_{n_{1} q_{1} p_{1} q_{2}} R_{n_{2} q_{1} p_{2} q_{2}}+\frac{1}{12} R_{n_{1} n_{2} p_{1} p_{2}} R_{m_{1} q_{1} p_{1} q_{2}} R_{m_{2} q_{1} p_{2} q_{2}}
\end{aligned}
$$




$$
\begin{array}{r}
-\frac{1}{10} g_{m_{2} n_{1}} R_{n_{2} r_{1} q_{1} q_{2}} R_{q_{1} q_{2} p_{1} p_{2}} R_{p_{1} p_{2} m_{1} r_{1}}+\frac{2}{5} g_{m_{2} n_{1}} R_{n_{2} q_{1} r_{1} q_{2}} R_{q_{1} p_{1} q_{2} p_{2}} R_{p_{1} m_{1} p_{2} r_{1}} \\
+\frac{1}{5} g_{m_{2} n_{1}} R_{n_{2} q_{1} m_{1} q_{2}} R_{q_{1} p_{1} p_{2} r_{1}} R_{q_{2} p_{1} p_{2} r_{1}} \\
+\frac{1}{120} g_{m_{2} n_{1}} g_{m_{1} n_{2}} R_{q_{1} q_{2} r_{1} r_{2}} R_{r_{1} r_{2} p_{1} p_{2}} R_{p_{1} p_{2} q_{1} q_{2}}-\frac{1}{30} g_{m_{2} n_{1}} g_{m_{1} n_{2}} R_{q_{1} r_{1} q_{2} r_{2}} R_{r_{1} p_{1} r_{2} p_{2}} R_{p_{1} q_{1} p_{2} q_{2}} .
\end{array}
$$

The two-index $\check{W}^{i}$ are symmetric traceless:

$$
\begin{aligned}
& \check{W}_{m_{1} n_{1}}^{1}=R_{m_{1} q_{1} q_{2} n_{2}} R_{q_{2} n_{2} p_{1} p_{2}} R_{p_{1} p_{2} n_{1} q_{1}}-\frac{1}{7} g_{m_{1} n_{1}} R_{q_{1} q_{2} r_{1} r_{2}} R_{r_{1} r_{2} p_{1} p_{2}} R_{p_{1} p_{2} q_{1} q_{2}}, \\
& \check{W}_{m_{1} n_{1}}^{2}=R_{m_{1} q_{2} q_{1} n_{2}} R_{q_{2} p_{1} n_{2} p_{2}} R_{p_{1} n_{1} p_{2} q_{1}}-\frac{1}{7} g_{m_{1} n_{1}} R_{q_{1} r_{1} q_{2} r_{2}} R_{r_{1} p_{1} r_{2} p_{2}} R_{p_{1} q_{1} p_{2} q_{2}}, \\
& \check{W}_{m_{1} n_{1}}^{3}=R_{m_{1} q_{2} n_{1} p_{1}} R_{q_{2} n_{2} q_{1} q_{2}} R_{p_{1} n_{2} q_{1} q_{2}} .
\end{aligned}
$$

We have two scalars $\check{S}^{i}$ :

$$
\begin{aligned}
& \check{S}^{1}=R_{m_{1} m_{2} n_{1} n_{2}} R_{n_{1} n_{2} p_{1} p_{2}} R_{p_{1} p_{2} m_{1} m_{2}}, \\
& \check{S}^{2}=R_{m_{1} n_{1} m_{2} n_{2}} R_{n_{1} p_{1} n_{2} p_{2}} R_{p_{1} m_{1} p_{2} m_{2}} .
\end{aligned}
$$

We also need the six-index $\check{V}^{i}$, which have a set of five antisymmetric indices, vanish if the six indices are antisymmetrised, and are traceless:

$$
\begin{aligned}
& \check{V}_{m_{1} n_{1} n_{2} n_{3} n_{4} n_{5}}^{1}=R_{p_{1} m_{1} n_{2} n_{3}} R_{p_{1} n_{4} q_{1} q_{2}} R_{n_{5} n_{1} q_{1} q_{2}} \\
& \quad-\frac{1}{3} g_{m_{1} n_{2}} R_{p_{1} p_{2} n_{5} n_{1}} R_{q_{1} q_{2} n_{3} n_{4}} R_{p_{1} p_{2} q_{1} q_{2}}+\frac{2}{3} g_{m_{1} n_{2}} R_{p_{1} p_{2} n_{5} n_{1}} R_{q_{1} q_{2} p_{1} n_{3}} R_{q_{1} q_{2} p_{2} n_{4}}, \\
& \quad \check{V}_{m_{1} n_{1} n_{2} n_{3} n_{4} n_{5}}^{2}=R_{p_{1} q_{1} n_{3} n_{4}} R_{m_{1} q_{2} p_{1} n_{2}} R_{q_{1} q_{2} n_{5} n_{1}} \\
& \quad+\frac{1}{3} g_{m_{1} n_{2}} R_{p_{1} p_{2} n_{5} n_{1}} R_{q_{1} q_{2} p_{1} n_{3}} R_{q_{1} q_{2} p_{2} n_{4}}-\frac{2}{3} g_{m_{1} n_{2}} R_{q_{1} q_{2} n_{5} n_{1}} R_{p_{1} q_{1} p_{2} n_{3}} R_{p_{2} q_{2} p_{1} n_{4}},
\end{aligned}
$$

and three 4-forms $\check{T}^{i}$ :

$$
\begin{aligned}
& \check{T}_{n_{1} n_{2} n_{3} n_{4}}^{1}=R_{p_{1} p_{2} n_{1} n_{2}} R_{q_{1} q_{2} n_{3} n_{4}} R_{p_{1} p_{2} q_{1} q_{2}}, \\
& \check{T}_{n_{1} n_{2} n_{3} n_{4}}^{2}=R_{p_{1} p_{2} n_{1} n_{2}} R_{q_{1} q_{2} p_{1} n_{3}} R_{q_{1} q_{2} p_{2} n_{4}}, \\
& \check{T}_{n_{1} n_{2} n_{3} n_{4}}^{3}=R_{q_{1} q_{2} n_{1} n_{2}} R_{p_{1} q_{1} p_{2} n_{3}} R_{p_{2} q_{2} p_{1} n_{4}} .
\end{aligned}
$$

The eleven-dimensional terms thus decompose as

$$
\begin{aligned}
X_{m_{1} m_{2} n_{1} n_{2}}^{5}= & \check{X}_{m_{1} m_{2} n_{1} n_{2}}^{5}-\frac{8}{45} g_{m_{2} n_{1}} \check{W}_{m_{1} n_{2}}^{1}-\frac{1}{315} g_{m_{2} n_{1}} g_{m_{1} n_{2}} \check{S}^{1}, \\
X_{m_{1} m_{2} n_{1} n_{2}}^{6}= & \check{X}_{m_{1} m_{2} n_{1} n_{2}}^{6}+\frac{2}{45} \check{W}_{m_{1} n_{2}}^{1}-\frac{8}{45} \check{W}_{m_{1} n_{2}}^{2}-\frac{4}{45} \check{W}_{m_{1} n_{2}}^{3} \\
& +\frac{1}{1260} g_{m_{2} n_{1}} g_{m_{1} n_{2}} \check{S}^{1}-\frac{1}{315} g_{m_{2} n_{1}} g_{m_{1} n_{2}} \check{S}^{2}, \\
V_{m_{1} n_{1} n_{2} n_{3} n_{4} n_{5}}^{2}= & \check{V}_{m_{1} n_{1} n_{2} n_{3} n_{4} n_{5}}^{2}-\frac{10}{21} g_{m_{1} n_{1}} \check{T}_{n_{2} n_{3} n_{4} n_{5}}^{2}+\frac{8}{21} g_{m_{1} n_{1}} \check{T}_{n_{2} n_{3} n_{4} n_{5}}^{3}, \\
W_{m_{1} n_{1}}^{1}= & \check{W}_{m_{1} n_{1}}^{1}+\frac{4}{77} g_{m_{1} n_{1}} \check{S}^{1}, \\
W_{m_{1} n_{1}}^{2}= & \check{W}_{m_{1} n_{1}}^{2}+\frac{4}{77} g_{m_{1} n_{1}} \check{S}^{2}, \\
W_{m_{1} n_{1}}^{3}= & \check{W}_{m_{1} n_{1}}^{3}, \\
T_{n_{1} n_{2} n_{3} n_{4}}^{i}= & \check{T}_{n_{1} n_{2} n_{3} n_{4}}^{i}, \quad S^{i}=\check{S}^{i} .
\end{aligned}
$$




\section{B More general solution}

In section 4.2.1 we were able to find a "minimal" solution to the M-theory Lichnerowicz, in the sense that we did not utilise all the possible terms listed in table 5 in the construction (4.9) of the supersymmetry operator $D_{a}$. Let us now consider adding the remaining terms of the table and see if we can find more solutions:

$$
\begin{aligned}
D^{\prime}: S \rightarrow & T^{*} \otimes S, \\
D_{a}^{\prime} \varepsilon= & D_{a} \varepsilon+\sum_{i=1}^{2} s_{i} \nabla_{a}\left(S^{i} \varepsilon\right)+\sum_{i=1}^{3} w_{i}\left(\frac{9}{10} \nabla^{b}\left(W_{a b}^{i} \varepsilon\right)-\frac{1}{10} \gamma_{a}^{b} \nabla^{c}\left(W_{c b}^{i} \varepsilon\right)+\gamma^{b_{1} b_{2}} \nabla_{b_{1}}\left(W_{a b_{2}}^{i} \varepsilon\right)\right) \\
& +\sum_{i=1}^{3} z_{i} \gamma^{c_{1} \ldots c_{4}} \nabla^{b}\left(Z_{a b c_{1} \ldots c_{4}}^{i} \varepsilon\right) \\
& +\sum_{i=1}^{3} u_{i}\left(-\frac{1}{2} \gamma^{b_{2} b_{3}} \nabla^{b_{1}}\left(U_{a b_{1} b_{2} b_{3}}^{i} \varepsilon\right)+\frac{3}{10} \gamma^{b_{2} b_{3}} \nabla^{b_{1}}\left(U_{b_{1} a b_{2} b_{3}}^{i} \varepsilon\right)\right. \\
& \left.\quad-\frac{2}{105} \gamma_{a}^{b_{1} b_{2} b_{3}} \nabla^{c}\left(U_{c b_{1} b_{2} b_{3}}^{i} \varepsilon\right)+\frac{1}{21} \gamma^{b_{1} b_{2} b_{3} b_{4}} \nabla_{b_{4}}\left(U_{a b_{1} b_{2} b_{3}}^{i} \varepsilon\right)\right),
\end{aligned}
$$

and

$$
\begin{aligned}
\tilde{D}^{\prime}: T^{*} \otimes S \rightarrow & S \\
\tilde{D}^{\prime a} \psi_{a}= & \tilde{D}^{a} \psi_{a}+\sum_{i=1}^{2} s_{i} \gamma^{a b} S^{i} \nabla_{a} \psi_{b}+\sum_{i=1}^{3} w_{i} \gamma^{a c} W_{a b}^{i}\left(\nabla_{c} \psi^{b}-\nabla^{b} \psi_{c}\right) \\
& +\sum_{i=1}^{3} z_{i} \gamma^{c_{1} \ldots c_{4}} Z_{a b c_{1} \ldots c_{4}}^{i} \nabla^{b} \psi^{a} \\
& +\sum_{i=1}^{3} u_{i}\left(\gamma^{a c_{3}} U_{a c_{1} c_{2} c_{3}}^{i} \nabla^{c_{1}} \psi^{c_{2}}-\frac{1}{21} \gamma^{b c_{1} c_{2} c_{3}} U_{a c_{1} c_{2} c_{3}}^{i}\left(\nabla^{a} \psi_{b}-\nabla_{b} \psi^{a}\right)\right) .
\end{aligned}
$$

As previously mentioned, the $S^{i}$ and $W^{i}$ terms do not actually contribute to anything at this level, so their coefficients will be unconstrained. We get that the 2 -form in the M-theory Lichnerowicz vanishes if

$$
z_{1}=-\frac{1}{14} u_{2}+\frac{3}{28} z_{2}+\frac{3}{2} z_{3},
$$

while eliminating the 4 -forms is solved by:

$$
\begin{aligned}
& x_{1}=-y_{2}-\frac{1}{36} u_{1}, \quad x_{7}=16 y_{2}, \quad t_{1}=-\frac{1}{81} u_{1}, \\
& x_{2}=16 y_{2}+\frac{2}{9} u_{1}, \quad x_{8}=16 y_{2}, \quad t_{2}=\frac{2}{135}\left(216 y_{2}-3 x_{5}+2 u_{1}\right), \\
& x_{3}=-2 y_{2}-\frac{1}{18} u_{1}, \quad y_{1}=-\frac{1}{4} y_{2}-\frac{1}{144} u_{1}, \quad t_{3}=-\frac{4}{405}\left(648 y_{2}-9 x_{5}+u_{1}\right), \\
& x_{4}=-16 y_{2}, \quad u_{2}=0, \quad v_{1}=-\frac{5}{36} u_{1}, \\
& x_{6}=-4 x_{5}+\frac{4}{9} u_{1}, \quad u_{3}=-u_{1}, \quad v_{2}=\frac{5}{72}\left(-216 y_{2}+45 x_{5}-5 u_{1}\right) \text {, }
\end{aligned}
$$


such that in total we have, in addition to $y_{2}$ and $x_{5}$, that $u_{1}, z_{2}, z_{3}, w_{i}, s_{i}$ remain undetermined. The resulting scalars in the Lichnerowicz are

$$
\begin{aligned}
\left.(\tilde{D} D \varepsilon)\right|_{\text {scalar }}= & -\frac{1}{4} \mathcal{R}+\frac{1}{2} x_{5}\left(R_{46}-4 A_{7}\right) \\
& -\frac{1}{2} \frac{1}{12} y_{2}\left(192 R_{41}+384 R_{42}+24 R_{43}+12 R_{44}-192 R_{45}-384 A_{7}\right) \\
& +\frac{1}{2} \frac{1}{144} u_{1}\left(-2 R_{43}-R_{44}+8 R_{45}+16 A_{7}\right),
\end{aligned}
$$

and the 8-form is

$$
\left.(\tilde{D} D \varepsilon)\right|_{8 \text {-form }}=-\frac{1}{4} y_{2}\left(-\frac{1}{4} R \cdot Y^{1}+R \cdot Y^{2}\right)+\frac{1}{4} \frac{1}{144} u_{1} R \cdot Y^{1} .
$$

The coefficients $s_{i}, w_{i}, z_{i}$ do not contribute at all, and so the corresponding terms in the operators are completely superfluous at this given order and given our simplifications.

We see that the $u_{1}$ freedom corresponds to the remaining invariant mentioned in [34, $35,39]$, which here takes the form $I_{Y_{1}}+\frac{1}{24}\left(I_{X}-\frac{1}{8} I_{Z}\right)$. Differently from $I_{X} \pm \frac{1}{8} I_{Y}$ we do not expect this combination to lead to a full $N=2$ invariant. However, the emergence of an $N=1$ invariant in an eleven dimensional setting suggests the interesting possibility of extending the Lichnerowicz method to M-theory on manifolds with boundary, notably on the Horava-Witten interval.

Open Access. This article is distributed under the terms of the Creative Commons Attribution License (CC-BY 4.0), which permits any use, distribution and reproduction in any medium, provided the original author(s) and source are credited.

\section{References}

[1] N. Hitchin, Generalized Calabi-Yau manifolds, Quart. J. Math. 54 (2003) 281 [math.DG/0209099] [INSPIRE].

[2] M. Gualtieri, Generalized complex geometry, Ph.D. thesis, Oxford U., Oxford, U.K. (2003) [math.DG/0401221] [INSPIRE].

[3] M. Graña, R. Minasian, M. Petrini and A. Tomasiello, Supersymmetric backgrounds from generalized Calabi-Yau manifolds, JHEP 08 (2004) 046 [hep-th/0406137] [INSPIRE].

[4] M. Graña, R. Minasian, M. Petrini and A. Tomasiello, Generalized structures of $N=1$ vacua, JHEP 11 (2005) 020 [hep-th/0505212] [INSPIRE].

[5] M. Graña, R. Minasian, M. Petrini and A. Tomasiello, A scan for new $N=1$ vacua on twisted tori, JHEP 05 (2007) 031 [hep-th/0609124] [INSPIRE].

[6] A. Coimbra, C. Strickland-Constable and D. Waldram, Supersymmetric backgrounds and generalised special holonomy, Class. Quant. Grav. 33 (2016) 125026 [arXiv:1411.5721] [INSPIRE].

[7] A. Coimbra and C. Strickland-Constable, Supersymmetric backgrounds, the Killing superalgebra and generalised special holonomy, JHEP 11 (2016) 063 [arXiv:1606.09304] [INSPIRE]. 
[8] A. Coimbra and C. Strickland-Constable, Generalised structures for $N=1$ AdS backgrounds, JHEP 11 (2016) 092 [arXiv: 1504.02465] [INSPIRE].

[9] A. Coimbra, C. Strickland-Constable and D. Waldram, Supergravity as generalised geometry I: type II theories, JHEP 11 (2011) 091 [arXiv:1107.1733] [INSPIRE].

[10] A. Lichnerowicz, Spineurs harmonique (in French), C. R. Acad. Sci. Paris A 257 (1963) 7.

[11] J.-M. Bismut, A local index theorem for non Kähler manifolds, Math. Ann. 284 (1989) 681 [INSPIRE].

[12] A. Coimbra, R. Minasian, H. Triendl and D. Waldram, Generalised geometry for string corrections, JHEP 11 (2014) 160 [arXiv:1407.7542] [INSPIRE].

[13] E. Bergshoeff and M. de Roo, Supersymmetric Chern-Simons terms in ten-dimensions, Phys. Lett. B 218 (1989) 210 [INSPIRE].

[14] E.A. Bergshoeff and M. de Roo, The quartic effective action of the heterotic string and supersymmetry, Nucl. Phys. B 328 (1989) 439 [INSPIRE].

[15] A. Coimbra and R. Minasian, in progress.

[16] C.M. Hull, Generalised geometry for M-theory, JHEP 07 (2007) 079 [hep-th/0701203] [INSPIRE].

[17] P. Pires Pacheco and D. Waldram, M-theory, exceptional generalised geometry and superpotentials, JHEP 09 (2008) 123 [arXiv:0804.1362] [INSPIRE].

[18] A. Coimbra, C. Strickland-Constable and D. Waldram, $E_{d(d)} \times \mathbb{R}^{+}$generalised geometry, connections and M-theory, JHEP 02 (2014) 054 [arXiv: 1112.3989] [INSPIRE].

[19] A. Coimbra, C. Strickland-Constable and D. Waldram, Supergravity as generalised geometry II: $E_{d(d)} \times \mathbb{R}^{+}$and M-theory, JHEP 03 (2014) 019 [arXiv:1212.1586] [INSPIRE].

[20] K. Peeters, Introducing Cadabra: a symbolic computer algebra system for field theory problems, hep-th/0701238 [INSPIRE].

[21] K. Peeters, A field-theory motivated approach to symbolic computer algebra, Comput. Phys. Commun. 176 (2007) 550 [cs/0608005] [INSPIRE].

[22] E. Cremmer, B. Julia and J. Scherk, Supergravity theory in eleven-dimensions, Phys. Lett. B 76 (1978) 409 [InSPIRE].

[23] J.P. Gauntlett and S. Pakis, The geometry of D = 11 Killing spinors, JHEP 04 (2003) 039 [hep-th/0212008] [INSPIRE].

[24] D.J. Gross and E. Witten, Superstring modifications of Einstein's equations, Nucl. Phys. B 277 (1986) 1 [InSPIRE].

[25] M.T. Grisaru, A.E.M. van de Ven and D. Zanon, Two-dimensional supersymmetric $\sigma$-models on Ricci flat Kähler manifolds are not finite, Nucl. Phys. B 277 (1986) 388 [inSPIRE].

[26] N. Sakai and Y. Tanii, One loop amplitudes and effective action in superstring theories, Nucl. Phys. B 287 (1987) 457 [InSPIRE].

[27] I. Antoniadis, R. Minasian, S. Theisen and P. Vanhove, String loop corrections to the universal hypermultiplet, Class. Quant. Grav. 20 (2003) 5079 [hep-th/0307268] [INSPIRE].

[28] C. Vafa and E. Witten, A one loop test of string duality, Nucl. Phys. B 447 (1995) 261 [hep-th/9505053] [INSPIRE]. 
[29] M.J. Duff, J.T. Liu and R. Minasian, Eleven-dimensional origin of string-string duality: a one loop test, Nucl. Phys. B 452 (1995) 261 [hep-th/9506126] [INSPIRE].

[30] M.B. Green and P. Vanhove, D instantons, strings and M-theory, Phys. Lett. B 408 (1997) 122 [hep-th/9704145] [INSPIRE].

[31] B. Souères and D. Tsimpis, Action principle and the supersymmetrization of Chern-Simons terms in eleven-dimensional supergravity, Phys. Rev. D 95 (2017) 026013 [arXiv: 1612.02021] [INSPIRE].

[32] Y. Hyakutake and S. Ogushi, $R^{4}$ corrections to eleven dimensional supergravity via supersymmetry, Phys. Rev. D 74 (2006) 025022 [hep-th/0508204] [INSPIRE].

[33] Y. Hyakutake and S. Ogushi, Higher derivative corrections to eleven dimensional supergravity via local supersymmetry, JHEP 02 (2006) 068 [hep-th/0601092] [INSPIRE].

[34] K. Peeters, P. Vanhove and A. Westerberg, Supersymmetric higher derivative actions in ten-dimensions and eleven-dimensions, the associated superalgebras and their formulation in superspace, Class. Quant. Grav. 18 (2001) 843 [hep-th/0010167] [INSPIRE].

[35] K. Peeters, P. Vanhove and A. Westerberg, Supersymmetric $R^{4}$ actions and quantum corrections to superspace torsion constraints, in Noncommutative structures in mathematics and physics. Proceedings, NATO Advanced Research Workshop, Kiev, Ukraine, 24-28 September 2000, pg. 153 [hep-th/0010182] [INSPIRE].

[36] H. Lü, C.N. Pope, K.S. Stelle and P.K. Townsend, Supersymmetric deformations of $G_{2}$ manifolds from higher order corrections to string and M-theory, JHEP 10 (2004) 019 [hep-th/0312002] [INSPIRE].

[37] S. Lukic and G.W. Moore, Flux corrections to anomaly cancellation in M-theory on a manifold with boundary, hep-th/0702160 [INSPIRE].

[38] M.A.A. van Leeuwen, A.M. Cohen and B. Lisser, LiE, http://www-math.univ-poitiers.fr/ maavl/LiE/.

[39] M. de Roo, H. Suelmann and A. Wiedemann, The supersymmetric effective action of the heterotic string in ten-dimensions, Nucl. Phys. B 405 (1993) 326 [hep-th/9210099] [INSPIRE].

[40] P. Kaste, R. Minasian and A. Tomasiello, Supersymmetric M-theory compactifications with fluxes on seven-manifolds and G structures, JHEP 07 (2003) 004 [hep-th/0303127] [INSPIRE].

[41] M.D. Freeman and C.N. Pope, $\beta$-functions and superstring compactifications, Phys. Lett. B 174 (1986) 48 [inSPIRE].

[42] K. Becker, D. Robbins and E. Witten, The $\alpha^{\prime}$ expansion on a compact manifold of exceptional holonomy, JHEP 06 (2014) 051 [arXiv: 1404.2460] [INSPIRE].

[43] I. Antoniadis, S. Ferrara, R. Minasian and K.S. Narain, $R^{4}$ couplings in $M$ and type-II theories on Calabi-Yau spaces, Nucl. Phys. B 507 (1997) 571 [hep-th/9707013] [INSPIRE].

[44] J.T. Liu and R. Minasian, Higher-derivative couplings in string theory: dualities and the B-field, Nucl. Phys. B 874 (2013) 413 [arXiv: 1304.3137] [InSPIRE].

[45] E. Witten, String theory dynamics in various dimensions, Nucl. Phys. B 443 (1995) 85 [hep-th/9503124] [INSPIRE]. 